\title{
Surgery versus stereotactic radiotherapy for treatment of pulmonary metastases. A systematic review of literature
}

\author{
Francesco Londero' ${ }^{1}$ (D) , William Grossi ${ }^{(i D}$, Angelo Morelli ${ }^{1}$, Orlando Parise ${ }^{2}$, Gianluca \\ Masullo1, Cecilia Tetta ${ }^{3}$ iD, Ugolino Livi ${ }^{1}$, Jos G Maessen ${ }^{2}$ \& Sandro Gelsomino*,2 iD \\ ${ }^{1}$ Department of Cardiothoracic Surgery, S. Maria della Misericordia University Hospital, Udine, Italy \\ ${ }^{2}$ Cardiovascular Research Institute Maastricht, Maastricht, The Netherlands \\ ${ }^{3}$ Radiology Department, Rizzoli Institute, Bologna, Italy \\ *Author for correspondence: sandro.gelsomino@maastrichtuniversity.nl
}

\section{Future Science}

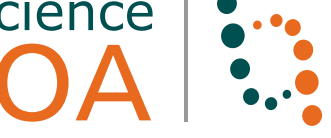

It is not clear as to which is the best treatment among surgery and stereotactic radiotherapy (SBRT) for lung oligometastases. A systematic review of literature with a priori selection criteria was conducted on articles on the treatment of pulmonary metastases with surgery or SBRT. Only original articles with a population of patients of more than 50 were selected. After final selection, 61 articles on surgical treatment and 18 on SBRT were included. No difference was encountered in short-term survival between pulmonary metastasectomy and SBRT. In the long-term surgery seems to guarantee better survival rates. Mortality and morbidity after treatment are $0-4.7 \%$ and $0-23 \%$ for surgery, and $0-2 \%$ and $4-31 \%$ for SBRT. Surgical metastasectomy remains the treatment of choice for pulmonary oligometastases.

Lay abstract: Patients with metastatic cancer with a limited number of deposits may benefit from surgical removal or irradiation of tumor nodules in addiction to chemotherapy. Surgical resection has been demonstrated to improve survival and, in some cases, can be curative. Stereotactic radiotherapy is emerging as a less invasive alternative to surgery, but settings and implications of the two treatments are profoundly different. The two techniques show similar results in the short-term, with lower complications rates for radiotherapy, while in the long-term surgery seems to guarantee higher survival rates.

First draft submitted: 27 September 2019; Accepted for publication: 16 February 2020; Published online: 15 April 2020

Keywords: lung • oligorecurrence $\bullet$ oligometastases $\bullet$ radiotherapy $\bullet$ surgery

Standard treatment for metastatic disease has historically been systemic chemotherapy, usually with only limited benefit on survival [1]. Evidence drawn from retrospective series have documented how patients with a limited pattern of disease demonstrate improved long-term outcomes when submitted to local aggressive treatments [2]. While complete surgical excision has always been acknowledged as the upfront treatment for this condition [3], in more recent years other techniques have been introduced, such as stereotactic radiotherapy (SBRT), which is a hypofractionated, high-dose delivery of radiation on a target tumor. This seems to guarantee comparable results with surgery in many retrospective series in terms of control of disease [4-6], with the advantage of lower invasiveness and postprocedural complications. To our knowledge, there is no prospective trial or systematic review to date comparing the effectiveness and safety of the two treatment modalities.

Therefore, we reviewed both retrospective and prospective case series, which assessed the efficacy of pulmonary metastasectomy or SBRT in patients with an oligometastatic state in terms of prognostic outcomes, control of disease and safety of treatment.

\section{Materials \& methods}

Search strategy

A literature search was conducted in accordance with the Preferred Reporting Items for Systematic Review and Metanalyses (PRISMA) [7]. An unrestricted literature search was performed using PubMed, EMBASE, Web of 
Science and Google Scholar Databases, as well as congress proceedings from major Thoracic and Cardiothoracic society meetings.

Search terms were: 'lung metastases' 'surgery' OR 'stereotactic radiotherapy.' The search strategy was decided by two authors (FL and WG) and approved by another reviewer (SG).

The literature was limited to articles published in English. References of original articles were reviewed manually and cross-checked for other relevant reports.

\section{Selection criteria \& quality assessment}

The literature research was conducted by previously defining a searching strategy using the PICOS method [8]. Initial selection criteria for study inclusion were:

- Type of study: original articles, no review or safety/feasibility studies;

- Primary tumor: no restrictions;

- Type of metastases: only pulmonary;

- Treatment modality: surgery or SBRT;

- Population $>50$;

- Adequate information regarding prognostic results, adverse events or both.

Since the first description of the oligometastatic state dates back to 1995, we included only articles published since 1995. Last search was run on 15th January 2018.

Two authors (FL and WG) selected the study for inclusion, extracted studies, as well as patient information and outcomes. Two reviewers (JGM and UL) independently assessed eligibility of the studies and risk of bias.

Studies assessing the efficacy of radiofrequency ablation were excluded since we decided to concentrate on the comparison between surgery and SBRT. If case sample size and/or treatment results have not been clearly reported in the abstract, articles were assessed via full text analysis for final inclusion in the review. In case of disagreement, consensus between reviewers was obtained through conjunct review and discussion throughout all the selection process.

We tried to contact one author to gather some information regarding results reported in his article [9], but we did not succeed in doing so.

Data were placed into a collection sheet and information included: characteristics of populations of patients (median age, sex ratio), inclusion/exclusion criteria of patients in the relative studies and basic disease (primary tumor, number of pulmonary lesions, diameter of lung deposits); interventions (surgery, SBRT or both) and treatment modalities (for surgery: surgical approach, kind of resection and postresectional status; for SBRT: radiation doses, number of fractions of treatment delivery); length of follow-up; outcomes of treatments (survival, rates of recurrence, progression-free survival [PFS], local control [LC], mortality, adverse events and surgical complications).

Articles were reviewed for risk of reporting biases or evident errors. Since both treatment modalities deal with a neoplastic condition the main outcome of interest was survival and incidence of recurrence. Where available we also collected data on the tolerability and safety of both treatments, in the sense of incidence of adverse events, complications and mortality within the first 30 days.

Given the wide heterogeneity of reporting clinical information across the different studies, we processed some data in order to get them aligned and comparable: gender prevalence has been modified into male/female number; age has been reported as it was originally as median and interquartile range, median and overall range or mean \pm standard deviation; primary tumor histology, type of treatment (approach, kind of resection and postresection status for surgery and dose of radiation and number of frames for SBRT), outcomes, morbidity, mortality and complications have been translated, where necessary, into percentages instead of absolute values.

\section{Results}

\section{Study selection}

Article selection process is reported in the diagram in Figure 1. The first search on electronic databases, after exclusion of duplicates, led to 4746 results. The first screening led to 685 abstracts, which were further scrutinized: 145 original works were selected based on language (only English), sample size ( $>50$ patients), pattern of disease (oligometastatic) and treatment performed with curative intent (SBRT and surgery, no radiofrequency ablation, 


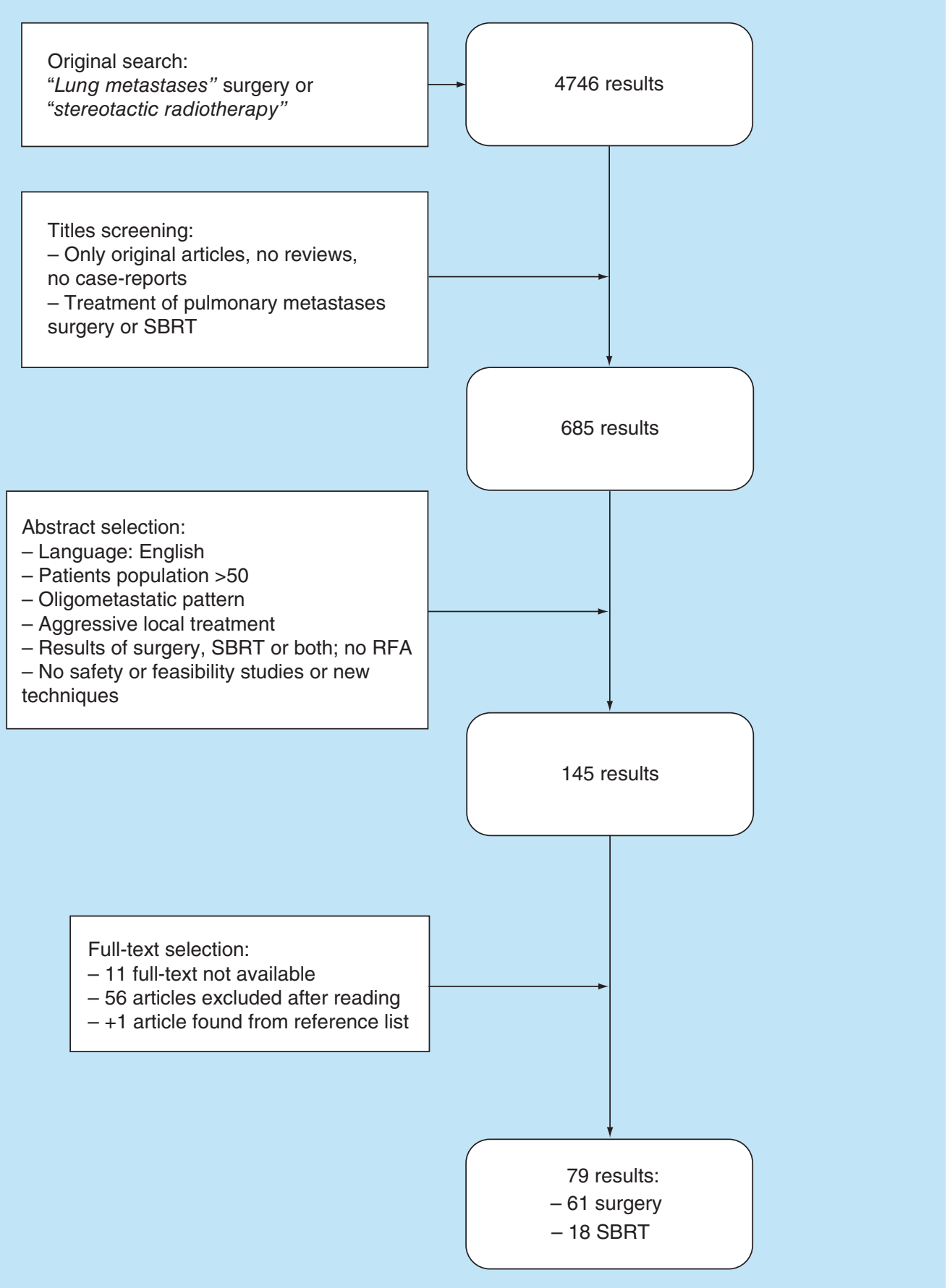

Figure 1. Flowchart of articles selection process.

RFA: Radiofrequency ablation; SBRT: Surgery and stereotactic radiotherapy.

cryoablation or other local treatments); safety or feasibility studies on new techniques were also excluded. If a manuscript reported on comparative results between surgical metastasectomy and SBRT, but only one of the two populations of study was greater than 50 patients, data were collected only on the arm of treatment meeting the minimal requirements on sample size. Last selection step was performed on full text analysis. One study was identified after checking references lists and included in the review. After final selection, a total of 79 studies were included and: of these, 61 were reports on surgical case series and 18 reported on the results of SBRT. 


\section{Bias}

The majority of surgical studies (50.8\%) do not report the size of resected lesions, which is a well-known prognostic factor and an important information regarding the burden of disease. Moreover, a few included articles reported the number of metastases ranging over five, which is the original uplimit for the definition of an oligometastatic state. Conversely, a minority of studies reported information regarding a history of previous local ablative treatments for oligometastases or the synchronous/metachronous development of metastases. Considering the relevant impact of these aspects on the patients' prognosis [10], this undoubtedly represents a huge limitation of the analysis. We observed how a relevant proportion of publications on surgical series report percentages of incomplete resections ranging between 3 and 21.3\%: this represents evidence of involved margins of resection in most cases, but some studies reported percentages of macroscopic residual disease. Including this subset of patients in survival analysis may have a considerable impact on survival rates of the whole population, considering the known detrimental effect of incomplete resection on prognosis [11].

None of the included studies on SBRT reported that a histological confirmation of the metastatic nature of nodules was carried out before or after treatment. This might suggest that a proportion of the irradiated nodules were not neoplastic, which has potential impact on the survival and recurrence analyses.

Although a statistical analysis on the difference of populations' age between surgical and SBRT series could not be performed, median/mean age tend to be higher in SBRT studies. This point should be considered when comparing results of both treatments, since populations might be different in terms of baseline conditions, with relevant effects on outcomes.

\section{Patient population \& tumor characteristics Surgery}

Results concerning study populations and the characteristics of tumors in surgical series are reported in Table 1. Among the 68 manuscripts on surgical series, only one was a prospective observational study, whereas the others were all retrospective. Some works report how their data were derived from prospectively maintained databases, but none of them clearly reported a prospective protocol for their study.

Population size ranged between 50 and 5206, with a median sample size of 114 patients (interquartile range [IQR]: 78-202). Apart from few articles reporting outcomes on the treatment of patients with metastases from sarcoma - where subjects were usually younger - mean or median values ranged uniformly between 48 and 66 years.

With the obvious exception of the series of patients treated for metastases from breast cancer, the majority of works reported a marked prevalence of males in their populations. The larger proportion of studies pertained to populations of patients with metastases from colorectal cancer only ( 24 articles) or various primary tumor origin (19 articles). Seven articles illustrated results of patients treated for metastases from primary sarcoma. The large majority of articles (95.1\%) report number of metastases, but the way this information is detailed vary quite extensively across studies: number of pulmonary deposits is referred as mean/patient, total number, single versus multiple or stratified per number with relative percentages/absolute numbers. The same observation can be made regarding size of pulmonary metastases, but in this case the information is provided in less than half of the manuscripts. A quite high conformity has been found in reported inclusion criteria. However, in 14 articles $(20.6 \%)$, no reference was made to criteria for enrolling patients in the studies [12-25].

\section{SBRT}

Information regarding the study population and tumor characteristics of SBRT series are reported in Table 2. With the exception of one prospective study, all included papers regarding the results of SBRT were retrospective case series. Median sample population was 66 patients (IQR: 61-96, range: 50-700). Age has usually been reported as median or mean and range, whereas in three articles it has been stratified as 60 years the cutoff for subdivision. Only in one article median age was $<60$ years [26], whereas most populations' median/mean age ranged between 65 and 71. Male sex was prevalent in the majority of articles (88.9\%) with a male/female ratio ranging between 0.93 and 6.12. Regarding tumor characteristics, $77.8 \%$ of articles included patients with pulmonary metastases arising from various primary tumors. Three articles $(16.7 \%)$ were focused on results of irradiated metastases from colorectal cancer and only one on the recurrence of lung tumors. The number of lung deposits has been presented as absolute numbers, mean/patient or stratified with relative number/percentages. Tumor size has been variously described as diameter or volume, this latter both as gross tumor volume (GTV) or planning tumor volume (PTV). In 22.2\% of papers, tumor size has been reported as stratified in classes, in a quite heterogeneous way across studies. Patients' 
Table 1. Surgery: study populations' and tumors' characteristics.

\begin{tabular}{|c|c|c|c|c|c|c|c|c|}
\hline Study (year) & Type & Pat & Age (range) & M:F & Primary tumor & Lesions ( $n$ ) & Tumor diameter & Ref. \\
\hline $\begin{array}{l}\text { Fourquier et al. } \\
\text { (1997) }\end{array}$ & $\mathrm{R}$ & 50 & $59 \ddagger(40-78)$ & $38: 12$ & $\mathrm{RCC}$ & 134 & ns & [12] \\
\hline $\begin{array}{l}\text { Robert et al. } \\
\text { (1997) }\end{array}$ & $\mathrm{R}$ & 276 & $38^{\ddagger}(5-78)$ & 193:83 & S: $46 \%$, T: $32 \%$, C: $19 \%$, M: $1.8 \%$, O: $1.4 \%$ & $1: 42.7 \% ;>1: 57.3 \%$ & ns & [44] \\
\hline $\begin{array}{l}\text { Pastorino et al. } \\
\text { (1997) }\end{array}$ & $\mathrm{R}$ & 5206 & $46^{\ddagger}(2-93)$ & 2932:2273 & E: $43 \%$, S: $42 \%$, GCC: $7 \%$, M: $6 \%$, O: $2 \%$ & $1: 45.8 \% ;>1: 54.2 \%$ & ns & [10] \\
\hline $\begin{array}{l}\text { van der Veen } \\
\text { et al. (1998) }\end{array}$ & $\mathrm{R}$ & 78 & $51^{\ddagger}(17-73)$ & $46: 32$ & $\begin{array}{l}\text { A: } 26.9 \%, \text { S: } 24.4 \% \text {, Te: } 19.2 \%, \text { RCC: } \\
16.7 \%, \text { M: } 12.8 \%\end{array}$ & ns & ns & [13] \\
\hline $\begin{array}{l}\text { Abecasis et al. } \\
\text { (1999) }\end{array}$ & $\mathrm{R}$ & 85 & $48.5^{\ddagger}(16-79)$ & $41: 44$ & $\begin{array}{l}\text { STS: } 21.2 \%, \text { Os: } 15.3 \%, \mathrm{HN}: 14.1 \%, \mathrm{~B}: \\
11.8 \%, \text { CRC: } 8.2 \%, \text { CC: } 5.9 \%, \mathrm{M}: 4.7 \% \text {, } \\
\text { RCC: } 3.5 \%, \mathrm{BI}: 3.5 \%, \mathrm{O}: 11.8 \%\end{array}$ & 1: $74.1 \% ;>1: 25.9 \%$ & ns & [45] \\
\hline $\begin{array}{l}\text { Kolodziejski } \\
\text { et al. (1999) }\end{array}$ & $\mathrm{R}$ & 93 & $50^{\dagger}(17-71)$ & 49:44 & $\begin{array}{l}\text { RCC: } 25 \%, \text { CRC: } 11 \%, \text { STS: } 11 \%, \text { M: } 10 \% \text {, } \\
\text { B: } 10 \%, \text { U: } 7 \%, \text { NSCLC: } 6 \%, \text { Te: } 4 \%, \text { O: } \\
16 \%\end{array}$ & $\begin{array}{l}1: 86 \% ; 2: 8,6 \% ; 3: \\
5.4 \%\end{array}$ & ns & [35] \\
\hline Leo et al. (2000) & $\mathrm{R}$ & 282 & $47^{\dagger}$ & $156: 126$ & M & $1: 65.1 \% ;>1: 34.9 \%$ & ns & [46] \\
\hline $\begin{array}{l}\text { Friedel et al. } \\
\text { (2001) }\end{array}$ & $\mathrm{R}$ & 467 & $53^{\ddagger}(21-87)$ & All $\mathrm{F}$ & B & $1: 65.9 \% ;>1: 44.1 \%$ & ns & [47] \\
\hline Piltz et al. (2002) & $\mathrm{R}$ & 105 & $59 \ddagger(35-78)$ & $73: 32$ & $\mathrm{RCC}$ & $1: 46.7 \% ;>153.3 \%$ & ns & [48] \\
\hline $\begin{array}{l}\text { Planchard et al. } \\
\text { (2003) }\end{array}$ & $\mathrm{R}$ & 125 & $53 \ddagger(30-82)$ & All $F$ & B & $1: 59.2 \% ;>1: 40.8 \%$ & $20.5^{\dagger}(5-70)$ & [49] \\
\hline $\begin{array}{l}\text { Monteiro et al. } \\
\text { (2004) }\end{array}$ & $\mathrm{R}$ & 78 & $59 \ddagger(25-78)$ & $41: 37$ & $\begin{array}{l}\text { Co: } 24.4 \%, \text { Re: } 20.5 \%, \text { B: } 15.4 \%, \text { U: } 7.7 \% \text {, } \\
\text { HN: } 6.4 \% \text {, UT: } 7.7 \% \text {, Te: } 3.8 \% \text {, O: } 14.1 \%\end{array}$ & $1: 67.9 \% ;>1: 32.1 \%$ & $\begin{array}{l}<30: 64.1 \% \\
>30: 35.9 \%\end{array}$ & [36] \\
\hline $\begin{array}{l}\text { Vogelsang et al. } \\
\text { (2004) }\end{array}$ & $\mathrm{R}$ & 75 & $58^{\ddagger}(33-82)$ & $42: 33$ & CRC & $1: 65.6 \% ;>1: 34.4 \%$ & $20 \ddagger(5-80)$ & [50] \\
\hline $\begin{array}{l}\text { Shiono et al. } \\
\text { (2005) }\end{array}$ & $\mathrm{R}$ & 87 & $61 \ddagger(23-82)$ & $57: 30$ & CRC & $1: 75.9 \% ;>1: 24.1 \%$ & $18^{\ddagger}(7-6.7)$ & [51] \\
\hline $\begin{array}{l}\text { Casali et al. } \\
\text { (2006) }\end{array}$ & $\mathrm{R}$ & 142 & $60.9(33-89)$ & $74: 68$ & $\begin{array}{l}\text { CRC: } 44 \%, \text { RCC: } 22 \%, \text { B: } 16 \%, \mathrm{HN}: 5 \%, \mathrm{U}: \\
4 \%, \text { SG: } 3 \%, \mathrm{BI}: 2 \%, \mathrm{O}: 4 \%\end{array}$ & $1: 65 \% ;>1: 35 \%$ & ns & [52] \\
\hline $\begin{array}{l}\text { Furak et al. } \\
\text { (2006) }\end{array}$ & $\mathrm{R}$ & 52 & $60.5^{\dagger}(20-86)$ & $32: 20$ & $\begin{array}{l}\text { CRC: } 37 \%, \text { RCC } 19 \%, \text { M: } 15 \%, \text { B: } 6 \%, \text { BI: } \\
6 \%, \text { U: } 4 \% \text {, Os: } 4 \%, \text { Te: } 2 \%, \text { SG: } 2 \%, \text { O: } \\
2 \% \text {, Th: } 2 \% \text {, L: } 2 \%\end{array}$ & $1: 82.7 \% ;>1: 17.3 \%$ & ns & [53] \\
\hline $\begin{array}{l}\text { Harting et al. } \\
(2006)\end{array}$ & $\mathrm{R}$ & 93 & $\begin{array}{l}<10: 17.2 \% \\
10-21: 82.8 \%\end{array}$ & ns & $S$ & $1: 28.3 \% ;>1: 71.7 \%$ & ns & [54] \\
\hline $\begin{array}{l}\text { Rena et al. } \\
(2006)\end{array}$ & $\mathrm{R}$ & 202 & $64 \ddagger(24-80)$ & 107:95 & $\begin{array}{l}\text { CRC: } 46.4 \%, \text { RCC: } 15.2 \% \text { B: } 11.4 \%, \mathrm{BI}: \\
10.2 \%, \mathrm{HN}: 8.2 \%, \text { U: } 4.3 \%, \text { O: } 4.3 \%\end{array}$ & $1: 72.7 \% ;>1: 27.3 \%$ & ns & [55] \\
\hline $\begin{array}{l}\text { Assouad et al. } \\
(2007)\end{array}$ & $\mathrm{R}$ & 65 & $62.1^{\dagger}(42-82)$ & $52: 13$ & $\mathrm{RCC}$ & ns & ns & [15] \\
\hline $\begin{array}{l}\text { Melloni et al. } \\
(2007)\end{array}$ & $\mathrm{R}$ & 81 & $61 \ddagger(38-83)$ & $49: 32$ & CRC & $1: 54.3 \% ;>1: 45.7 \%$ & $20^{\ddagger}(10-130)$ & {$[16]$} \\
\hline $\begin{array}{l}\text { Veronesi et al. } \\
\text { (2007) }\end{array}$ & $\mathrm{R}$ & 124 & $59 \ddagger(24-82)$ & $61: 63$ & $\begin{array}{l}\text { C: } 43.5 \%, \text { S: } 12.1 \%, \text { HN: } 6.4 \%, \text { UT: } 12.9 \% \text {, } \\
\text { B: } 8.9 \%, \text { Gy: } 6.4 \%, \text { M: } 4.8 \%, \text { GCC: } 2.4 \% \text {, } \\
\text { O: } 2.4 \%\end{array}$ & 1: $58.9 \% ;>141.1 \%$ & ns & [56] \\
\hline $\begin{array}{l}\text { Dahabre et al. } \\
(2008)\end{array}$ & $\mathrm{R}$ & 57 & $60 \ddagger(30-87)$ & $34: 23$ & CRC & $\begin{array}{l}\text { 1: } 59.65 \% ;>1: \\
40.35 \%\end{array}$ & ns & [9] \\
\hline $\begin{array}{l}\text { Smith et al. } \\
\text { (2008) }\end{array}$ & $\mathrm{R}$ & 94 & $49 \ddagger(9-75)$ & $47: 47$ & $\mathrm{~S}$ & $1: 36.2 \% ;>1: 63.8 \%$ & ns & [17] \\
\hline $\begin{array}{l}\text { Yoshimoto et al. } \\
\text { (2008) }\end{array}$ & $\mathrm{R}$ & 90 & $55.1^{\dagger}(32-77)$ & All $F$ & B & 1: $86.7 \% ;>1: 13.3 \%$ & $23^{\dagger}(8-48)$ & [37] \\
\hline $\begin{array}{l}\text { Winter et al. } \\
(2008)\end{array}$ & $\mathrm{R}$ & 67 & $57.1^{\dagger}$ & $53: 14$ & $\mathrm{HN}$ & $1: 79.1 \% ;>1: 20.9 \%$ & $\begin{array}{l}<30: 79.1 \% \\
>30: 20.9 \%\end{array}$ & [57] \\
\hline Lin et al. (2009) & $\mathrm{R}$ & 63 & $58.7^{\dagger}(32-78)$ & $39: 24$ & CRC & $1: 65 \% ;>1: 35 \%$ & $27.7^{\dagger}(5-86)$ & [58] \\
\hline \multicolumn{9}{|c|}{$\begin{array}{l}\text { Values expressed as median/mean (range): } \\
\text { †Mean. } \\
\text { ¥Median. } \\
\text { A: Adenocarcinoma; B: Breast; BI: Bladder; C: Carcinoma; CC: Cervical carcinoma; Co: Colon; CRC: Colorectal cancer; E: Epithelial; En: Endometrial; GCC: Germ-cell cancer; GI: } \\
\text { Gastrointestinal tract; Go: Gonads; Gy: Gynecological tract; HN: Head \& neck; IQR: Interquartile range; L: Larynx; M: Melanoma; M/F: Male/female; ns: Not stated; NSCLC: Non- } \\
\text { small-cell lung cancer; O: Other; Os: Osteosarcoma; Ov: Ovary; P: Prospective; Pa: Prostate; Pat: Number of patients; R: Retrospective; RCC: Renal cell carcinoma; Re: Rectum; S: } \\
\text { Sarcoma; SG: Salivary gland; STS: Soft tissue sarcoma; T: Teratoma; Te: Testicle; Th: Thyroid; Ty: Thymus; U: Uterine; UL: Uterine leyomiosarcoma; UT: Urinary tract; VATS: Video-assisted } \\
\text { thoracic surgery. }\end{array}$} \\
\hline
\end{tabular}


Systematic Review Londero, Grossi, Morelli et al.

Table 1. Surgery: study populations' and tumors' characteristics (cont.).

\begin{tabular}{|c|c|c|c|c|c|c|c|c|}
\hline Study (year) & Type & Pat & Age (range) & $M: F$ & Primary tumor & Lesions ( $n$ ) & Tumor diameter & Ref. \\
\hline $\begin{array}{l}\text { Rama et al. } \\
(2009)\end{array}$ & $\mathrm{R}$ & 61 & $61.2^{\dagger}(30-80)$ & $42: 19$ & CRC & $1: 60.6 \% ;>1: 39.4 \%$ & $\begin{array}{l}>30: 72 \% ;>30: \\
28 \%\end{array}$ & [59] \\
\hline $\begin{array}{l}\text { Takakura et al. } \\
\text { (2009) }\end{array}$ & $\mathrm{R}$ & 56 & $64^{\ddagger}(42-76)$ & $26: 30$ & CRC & $1: 68.5 \% ;>1: 31.5 \%$ & $20^{\ddagger}(5-88)$ & [60] \\
\hline $\begin{array}{l}\text { Borasio et al. } \\
(2010)\end{array}$ & $\mathrm{R}$ & 137 & $63.8^{\dagger}(35-82)$ & $82: 55$ & CRC & $1: 59.9 \% ;>1: 40.1 \%$ & $20^{\ddagger}(2.5-70.0)$ & [61] \\
\hline $\begin{array}{l}\text { Riquet et al. } \\
(2010)\end{array}$ & $\mathrm{R}$ & 127 & $65^{\dagger}(36-85)$ & $74: 53$ & CRC & $1: 59.0 \% ;>1: 41.0 \%$ & $30.2^{\dagger}(2-90)$ & [62] \\
\hline $\begin{array}{l}\text { Sardenberg } \\
\text { et al. (2010) }\end{array}$ & $\mathrm{R}$ & 77 & $\begin{array}{l}<45: 48.1 \% \\
>45: 51.9 \%\end{array}$ & $37: 40$ & $\mathrm{~S}$ & 1: $55.9 \% ;>1: 44.1 \%$ & $\begin{array}{l}<20: 27.4 \% \\
>20: 72.6 \%\end{array}$ & [63] \\
\hline $\begin{array}{l}\text { Tacconi et al. } \\
(2010)\end{array}$ & $\mathrm{R}$ & 87 & $60.5^{\dagger}(16-82)$ & $47: 40$ & $\begin{array}{l}\text { CRC: } 51.7 \%, \text { RCC: } 14.9 \%, \text { U: } 8 \%, \text { L: } 5.7 \% \text {, } \\
\text { B: } 3.4 \%, \text { Pa: } 2.3 \%, \text { Ov: } 2.3 \%, \text { S: } 8 \%, \text { M: } \\
3.4 \%\end{array}$ & 191 & ns & [64] \\
\hline $\begin{array}{l}\text { Casiraghi et al. } \\
\text { (2011) }\end{array}$ & $\mathrm{R}$ & 575 & $58^{\dagger}(14-83)$ & $247: 328$ & $\begin{array}{l}\text { E: } 75.8 \%, \text { GI: } 36.5 \%, \text { B: } 8.9 \%, \text { UT: } 14.9 \% \text {, } \\
\text { Gy: } 7.3 \%, \text { HN: } 7.5 \%, \text { Ty: } 0.7 \%, \text { S: } 16.3 \% \text {, } \\
\text { M: } 5.7 \%, \text { GCC: } 2.1 \%\end{array}$ & $1: 36.3 \% ;>1: 63.7 \%$ & ns & [65] \\
\hline $\begin{array}{l}\text { Long et al. } \\
\text { (2011) }\end{array}$ & $\mathrm{R}$ & 55 & $47 \ddagger(12-88)$ & $40: 15$ & $\begin{array}{l}\text { CRC: } 25.4 \%, \text { HCC: } 16.4 \%, \text { RCC: } 10.8 \% \text {, Os: } \\
5.5 \% \text {, NSCLC: } 5.5 \%, \text { HN: } 5.5 \%, \text { U: } 3.6 \% \text {, O: } \\
27.2 \%\end{array}$ & 174 & ns & [66] \\
\hline $\begin{array}{l}\text { Stephens et al. } \\
\text { (2011) }\end{array}$ & $\mathrm{R}$ & 81 & $43.5^{\ddagger}$ & $45: 36$ & $S$ & mean $5.5 /$ patient & ns & [67] \\
\hline $\begin{array}{l}\text { Zabaleta et al. } \\
\text { (2011) }\end{array}$ & $\mathrm{R}$ & 84 & $65^{\ddagger}(40-82)$ & $60: 24$ & CRC & $1: 77.4 \% ;>1: 22.6 \%$ & $\begin{array}{l}<20: 42.9 \% \\
>20: 57.1 \%\end{array}$ & [68] \\
\hline $\begin{array}{l}\text { Blackmon et al. } \\
\text { (2012) }\end{array}$ & $\mathrm{R}$ & 229 & $60 \ddagger(24-82)$ & $129: 100$ & CRC & $1: 76.9 \% ;>1: 23.1 \%$ & $15^{\ddagger}(2-145)$ & [18] \\
\hline $\begin{array}{l}\text { Chua et al. } \\
\text { (2012) }\end{array}$ & $\mathrm{R}$ & 292 & $59 \ddagger(19-84)$ & $208: 84$ & $M$ & $1: 59.2 \% ;>1: 40.8 \%$ & $20 \ddagger(10-150)$ & [69] \\
\hline $\begin{array}{l}\text { Goonerante } \\
\text { et al. (2012) }\end{array}$ & $\mathrm{R}$ & 106 & $64 \ddagger(I Q R: 16-46)$ & $59: 57$ & CRC & $1: 75.5 \% ;>1: 24.5 \%$ & $20 \ddagger(14-38)$ & [19] \\
\hline $\begin{array}{l}\text { Hirosawa et al. } \\
\text { (2012) }\end{array}$ & $\mathrm{R}$ & 266 & $64 \ddagger(25-86)$ & 168:98 & CRC & $1: 45.1 \% ;>1: 54.9 \%$ & $18^{\ddagger}(4-70)$ & [70] \\
\hline $\begin{array}{l}\text { Mizuno et al. } \\
(2012)\end{array}$ & $\mathrm{R}$ & 52 & $41^{\ddagger}(7-74)$ & $34: 18$ & $S$ & $1: 53.8 \% ;>1: 46.2 \%$ & ns & [20] \\
\hline $\begin{array}{l}\text { Lo Faso et al. } \\
\text { (2013) }\end{array}$ & $\mathrm{R}$ & 164 & $64.17^{\dagger} \pm 14.4$ & $100: 64$ & S: $17.1 \%$, M: $4.3 \%$, GCC: $1.8 \%$, E: $76.38 \%$ & $1: 59.1 \% ;>1: 40.9 \%$ & ns & [71] \\
\hline $\begin{array}{l}\text { Poletti et al. } \\
(2013)\end{array}$ & $\mathrm{R}$ & 119 & $52^{\ddagger}(15-75)$ & $68: 51$ & CRC: $47.9 \%$, S: $21.8 \%$, HN: $7.5 \%$, Go: $5.9 \%$ & 511 & ns & [38] \\
\hline Cho et al. (2014) & $\mathrm{R}$ & 626 & $62^{\ddagger}(23-86)$ & $390: 136$ & CRC & $1 \ddagger(1-21)$ & 16 & [72] \\
\hline $\begin{array}{l}\text { Renaud et al. } \\
\text { (2014) }\end{array}$ & $\mathrm{R}$ & 320 & $63^{\ddagger}(27-86)$ & 215:105 & CRC & $1: 49.4 \% ;>1: 50.6 \%$ & ns & [73] \\
\hline $\begin{array}{l}\text { Renaud et al. } \\
\text { (2014) }\end{array}$ & $\mathrm{R}$ & 122 & $63.3^{\dagger}(43-82)$ & $84: 38$ & $\mathrm{RCC}$ & $1: 35.2 \% ;>1: 64.8 \%$ & ns & [74] \\
\hline $\begin{array}{l}\text { Zampino et al. } \\
(2014)\end{array}$ & $\mathrm{R}$ & 199 & $61 \ddagger(19-82)$ & $125: 74$ & CRC & $1: 47.7 \% ;>1: 52.3 \%$ & ns & [75] \\
\hline $\begin{array}{l}\text { Booth et al. } \\
(2015)\end{array}$ & $\mathrm{R}$ & 709 & $65^{\dagger}$ & $424: 285$ & CRC & ns & ns & [41] \\
\hline Hou et al. (2015) & $\mathrm{R}$ & 114 & $62^{\ddagger}(45-76)$ & $74: 40$ & CRC & 151 & $40^{\ddagger}(10-70)$ & [21] \\
\hline $\begin{array}{l}\text { Seebacher et al. } \\
\text { (2015) }\end{array}$ & $\mathrm{R}$ & 209 & $62.4^{\dagger}(30-81)$ & $110: 99$ & $\begin{array}{l}\text { CRC: } 40.2 \%, \text { RCC: } 18.2 \% \text {, B: } 10.5 \%, \text { U: } \\
6.7 \%, \text { HN: } 4.8 \%, \text { M: } 4.3 \%, \text { S: } 3.8 \% \text {, Th: } \\
3.8 \%, \text { UT: } 3.3 \%, \text { O: } 4.8 \%\end{array}$ & $3.4^{\dagger} \pm 4.1$ per pat & $26^{\dagger}$ & [76] \\
\hline $\begin{array}{l}\text { Guerrera et al. } \\
(2016)\end{array}$ & $\mathrm{R}$ & 188 & $66 \ddagger(I Q R: 58-72)$ & $109: 79$ & CRC & $1: 53 \% ;>1: 47 \%$ & $30 \ddagger$ & [77] \\
\hline \multicolumn{9}{|c|}{$\begin{array}{l}\text { Values expressed as median/mean (range): } \\
\text { †Mean. } \\
\text { † Median. } \\
\text { A: Adenocarcinoma; B: Breast; BI: Bladder; C: Carcinoma; CC: Cervical carcinoma; Co: Colon; CRC: Colorectal cancer; E: Epithelial; En: Endometrial; GCC: Germ-cell cancer; GI: } \\
\text { Gastrointestinal tract; Go: Gonads; Gy: Gynecological tract; HN: Head \& neck; IQR: Interquartile range; L: Larynx; M: Melanoma; M/F: Male/female; ns: Not stated; NSCLC: Non- } \\
\text { small-cell lung cancer; O: Other; Os: Osteosarcoma; Ov: Ovary; P: Prospective; Pa: Prostate; Pat: Number of patients; R: Retrospective; RCC: Renal cell carcinoma; Re: Rectum; S: } \\
\text { Sarcoma; SG: Salivary gland; STS: Soft tissue sarcoma; T: Teratoma; Te: Testicle; Th: Thyroid; Ty: Thymus; U: Uterine; UL: Uterine leyomiosarcoma; UT: Urinary tract; VATS: Video-assisted } \\
\text { thoracic surgery. }\end{array}$} \\
\hline
\end{tabular}


Table 1. Surgery: study populations' and tumors' characteristics (cont.).

\begin{tabular}{|c|c|c|c|c|c|c|c|c|}
\hline Study (year) & Type & Pat & Age (range) & $M: F$ & Primary tumor & Lesions ( $\mathrm{n}$ ) & Tumor diameter & Ref. \\
\hline $\begin{array}{l}\text { Filippi et al. } \\
(2016)\end{array}$ & $\mathrm{R}$ & 142 & $66^{\ddagger}$ (IQR: 59-72) & $87: 55$ & CRC & $1: 54.9 \% ;>1: 45.1 \%$ & $15^{\ddagger}$ & [30] \\
\hline $\begin{array}{l}\text { Franzke et al. } \\
\text { (2016) }\end{array}$ & $\mathrm{R}$ & 178 & $59.3^{\dagger}(17-85.4)$ & $110: 68$ & $\begin{array}{l}\text { CRC: } 30.3 \%, \text { RCC: } 16.9 \%, \text { S: } 14.6 \%, \text { M: } \\
10.6 \%, \text { GCC: } 3.9 \% \text {, B: } 3.4 \%, \text { O: } 20.3 \%\end{array}$ & $1: 55.6 \% ;>1: 44.4 \%$ & $19.6^{\dagger}(3-120)$ & [78] \\
\hline $\begin{array}{l}\text { Girelli et al. } \\
(2016)\end{array}$ & $\mathrm{R}$ & 109 & $48^{\ddagger}$ (IQR: $\left.31-65\right)$ & $58: 51$ & SG & $1: 26.6 \% ;>1: 73.4 \%$ & ns & [79] \\
\hline $\begin{array}{l}\text { Okiror et al. } \\
(2016)\end{array}$ & $\mathrm{R}$ & 66 & $51^{\ddagger}(16-79)$ & $39: 27$ & $\mathrm{~S}$ & $3^{\ddagger}(1-9)$ & ns & [80] \\
\hline $\begin{array}{l}\text { Guerrini et al. } \\
\text { (2016) }\end{array}$ & $\mathrm{R}$ & 224 & $64^{\dagger} \pm 14.4$ & $140: 84$ & $\begin{array}{l}\text { S: } 12.5 \%, \text { M: } 3 \%, \text { GCC: } 1.3 \%, \text { CRC: } 59.8 \% \text {, } \\
\text { B: } 10.7 \%, \text { UT: } 5.8 \%, \text { Gy: } 0.9 \%, \text { HN: } 5.8 \%\end{array}$ & 1: $56.7 \%>1: 43.3 \%$ & ns & [39] \\
\hline $\begin{array}{l}\text { Gafencu et al. } \\
\text { (2017) }\end{array}$ & $\mathrm{R}$ & 327 & $50.57^{\dagger} \pm 16.21$ & $166: 161$ & $S$ & $\begin{array}{l}1-3: 86.5 \% ; 4+: \\
23.5\end{array}$ & $21.7^{\dagger}$ & [22] \\
\hline Kim et al. (2017) & $\mathrm{R}$ & 129 & $56^{\ddagger}(33-76)$ & $77: 52$ & CRC & 1: $78.3 \% ;>1: 21.7 \%$ & $\begin{array}{l}<10: 33.3 \% \\
>10: 66.7 \%\end{array}$ & [23] \\
\hline $\begin{array}{l}\text { Shiomi et al. } \\
(2017)\end{array}$ & $\mathrm{R}$ & 100 & $64.7^{\dagger} \pm 10.0$ & $56: 44$ & CRC & $1: 72 \% ;>1: 28 \%$ & $\begin{array}{l}<20: 68 \% ;>20: \\
32 \%\end{array}$ & [81] \\
\hline Sun et al. (2017) & $\mathrm{R}$ & 154 & $\begin{array}{l}<65: 71.4 \% \\
>65: 28.6 \%\end{array}$ & $101: 53$ & CRC & $1: 81.2 \% ;>1: 18.8 \%$ & $21^{\ddagger}$ & [82] \\
\hline $\begin{array}{l}\text { Shiono et al. } \\
(2017)\end{array}$ & $\mathrm{R}$ & 553 & $66^{\ddagger}(29-89)$ & $314: 139$ & CRC & $\begin{array}{l}\text { Median per pat: } 1 \\
(1-8)\end{array}$ & $15^{\ddagger}(5-51)$ & [14] \\
\hline $\begin{array}{l}\text { Lodeweges et al. } \\
\text { (2017) }\end{array}$ & $\mathrm{R}$ & 68 & $61 \ddagger(18-80)$ & $37: 31$ & CRC: $57.3 \%$, S: $26.5 \%$, RCC: $7.3 \%, 0: 8.8 \%$ & 109 (1.60 per pat) & $20^{\dagger}(1.7-2.4)$ & [24] \\
\hline \multicolumn{9}{|c|}{$\begin{array}{l}\text { Values expressed as median/mean (range): } \\
\text { †Mean. } \\
\text { ¥Median. } \\
\text { A: Adenocarcinoma; B: Breast; BI: Bladder; C: Carcinoma; CC: Cervical carcinoma; Co: Colon; CRC: Colorectal cancer; E: Epithelial; En: Endometrial; GCC: Germ-cell cancer; GI: } \\
\text { Gastrointestinal tract; Go: Gonads; Gy: Gynecological tract; HN: Head \& neck; IQR: Interquartile range; L: Larynx; M: Melanoma; M/F: Male/female; ns: Not stated; NSCLC: Non- } \\
\text { small-cell lung cancer; O: Other; Os: Osteosarcoma; Ov: Ovary; P: Prospective; Pa: Prostate; Pat: Number of patients; R: Retrospective; RCC: Renal cell carcinoma; Re: Rectum; S: } \\
\text { Sarcoma; SG: Salivary gland; STS: Soft tissue sarcoma; T: Teratoma; Te: Testicle; Th: Thyroid; Ty: Thymus; U: Uterine; UL: Uterine leyomiosarcoma; UT: Urinary tract; VATS: Video-assisted } \\
\text { thoracic surgery. }\end{array}$} \\
\hline
\end{tabular}

inclusion criteria were reported in $83.3 \%$ of articles, $72.2 \%$ also described definition criteria for an oligometastatic state and, in most of the publications, the maximum size of metastases accepted for irradiation $<50 \mathrm{~mm}$.

\section{Treatment}

Surgery

The description of surgical approaches and length of follow-up are presented in Table 3. A common attitude was determined regarding surgical approach and extent of resection. The most common surgical approach reported was thoracotomy. Interestingly, a positive trend has been found in more recent publications in the reported percentages of resection performed via thoracoscopy (video-assisted thoracic surgery [VATS]). Bilateral localizations of disease have been treated with different approaches: staged thoracotomies within a short interval of time represent the main proportion, while other approaches such as median sternotomy, synchronous bilateral thoracotomy, handassisted procedures and clamshell incision have been described in several papers. A large proportion of authors favored a lung-sparing resection, atypical resection, which was also the most frequent intervention performed. Wedge resections were reported as almost the totality of procedure performed in one paper employing a substernal hand-assisted approach, whereas they represent only $11 \%$ of resections performed in a series of patients with metastases from breast cancer. Apart from these cases, in many publications atypical resections have been employed in approximately $60-70 \%$ of surgical procedures. Extensive resections represent a minor proportion in the majority of works, but pneumonectomies constitute up to $19.5 \%$ of resections performed in some series. Several studies mention percentages of patients were residual disease could be assumed (microscopic, R1) or evident (macroscopic, R2) after metastasectomy. A R0 resection was achieved in more than $90 \%$ of cases in most articles, but rates of incomplete resection and even macroscopic residual disease could reach $20 \%$ in several reports [18].

There was wide heterogeneity in reporting follow-up time with 15 authors $(24.6 \%)$ not mentioning it at all. Median follow-up was greater than 2 years in $67.2 \%$ and greater than 4 years in $18 \%$ of included articles. 
Table 2. Stereotactic radiotherapy: studies' population and tumor characteristics.

\begin{tabular}{|c|c|c|c|c|c|c|c|c|}
\hline Study (year) & Type & Pat & Age (range) & $M: F$ & Primary tumor & Lesions ( $\mathrm{n}$ ) & Tumor diameter & Ref. \\
\hline Hof et al. (2007) & $\mathrm{R}$ & 61 & $65.9^{\dagger}(25-87)$ & $40: 21$ & $\begin{array}{l}\text { NSCLC: } 50.8 \% \text {; CRC: } 13.1 \% \text {; B: } \\
6.5 \% ; \text { O: } 29.5 \%\end{array}$ & 71 & GTV $10 \mathrm{~cm}^{3 \dagger}(1-53)$ & [6] \\
\hline $\begin{array}{l}\text { Ricardi et al. } \\
\text { (2011) }\end{array}$ & $\mathrm{R}$ & 61 & $70^{\dagger}(46-86)$ & $2.34: 1$ & $\begin{array}{l}\text { NSCLC: } 53.5 \% \text {; CRC: } 21.3 \% \text {; Pa: } \\
3.3 \% ; \text { HCC: } 3.3 \% \text {; HN: } 3.3 \% \text {; O: } \\
12.8 \%\end{array}$ & 77 & $20 \mathrm{~mm}^{\dagger}(7-45)$ & [83] \\
\hline $\begin{array}{l}\text { Zhang et al. } \\
\text { (2011) }\end{array}$ & $\mathrm{R}$ & 71 & $59^{\dagger}(15-84)$ & $45: 26$ & $\begin{array}{l}\text { NSCLC: } 18.3 \% \text {; CRC: } 15.5 \% \text {; HN: } \\
\text { 14.1\%; S: } 11.3 \% \text {; HCC: } 11.3 \% \text {; } \\
\text { RCC: } 8.5 \% ; B: 7.0 \% ; \text { O: } 14.1 \%\end{array}$ & 172 & $21 \mathrm{~mm}^{\dagger}(0.9-7.9)$ & [25] \\
\hline Oh et al. (2012) & $\mathrm{R}$ & 57 & $\begin{array}{l}<60: 28 \% \text {; >60: } \\
72 \%\end{array}$ & $49: 8$ & $\begin{array}{l}\text { NSCLC: } 49.2 \% \text {; HCC: } 13.4 \% \text {; CRC: } \\
\text { 10.5\%; HN: } 16.4 \% \text {; O: } 10.5 \%\end{array}$ & 67 & $\begin{array}{l}<25 \mathrm{~mm}: 86.6 \% \\
>25 \mathrm{~mm}: 13.4 \%\end{array}$ & [34] \\
\hline $\begin{array}{l}\text { Filippi et al. } \\
\text { (2014) }\end{array}$ & $\mathrm{R}$ & 67 & $71^{\dagger}(40-86)$ & $44: 31$ & $\begin{array}{l}\text { NSCLC: } 37.4 \% \text {; CRC: } 40.3 \% \text {; M: } \\
7.5 \% \text {; HN: } 4.5 \% \text {; HCC: } 2.9 \% \text {; Oe: } \\
2.9 \% ; B: 1.5 \% \text {; RCC: } 1.5 \% \text {; Pr: } \\
1.5 \%\end{array}$ & 90 & $17 \mathrm{~mm}^{\dagger}(7-38)$ & [5] \\
\hline Osti et al. (2013) & $\mathrm{P}$ & 66 & $68^{\dagger}(25-89)$ & $32: 34$ & $\begin{array}{l}\text { NSCLC: } 18 \% \text {; CRC: } 35 \% \text {; B: } 17 \% \text {; } \\
\text { O: } 30 \%\end{array}$ & 103 & $\begin{array}{l}<10 \text { cc: } 62 \% ;>10 \text { cc: } \\
38 \%\end{array}$ & [29] \\
\hline Siva et al. (2015) & $\mathrm{R}$ & 65 & $69^{\dagger}$ (IQR: 61-78) & $38: 27$ & $\begin{array}{l}\text { CRC: } 31 \% \text {; NSCLC: } 25 \% \text {; HN: } 11 \% \text {; } \\
\text { STS: } 8 \% ; \text { O: } 25 \%\end{array}$ & $\begin{array}{l}1: 78.5 \% ;>1: \\
21.5 \%\end{array}$ & ns & [84] \\
\hline $\begin{array}{l}\text { Aoki et al. } \\
(2015)\end{array}$ & $\mathrm{R}$ & 66 & $71^{\dagger}(27-87)$ & $44: 22$ & $\begin{array}{l}\text { NSCLC: } 47 \% \text {; CRC: } 19.7 \% \text {; HN: } \\
15.1 \% \text {; Oe: } 4.5 \% \text {; U: } 4.5 \% \text {; O: } \\
9.1 \%\end{array}$ & 76 & $\leq 30: 92.1 \% ;>30: 7.9 \%$ & [85] \\
\hline $\begin{array}{l}\text { Binkley et al. } \\
\text { (2015) }\end{array}$ & $\mathrm{R}$ & 77 & $60^{\dagger}(30-93)$ & $40: 37$ & $\begin{array}{l}\text { NSCLC: } 17.2 \% \text {; CRC: } 21.3 \% ; \text { S: } \\
15.6 \% ; 0: 45.9 \%\end{array}$ & 122 & GTV: $3.7 \mathrm{ml}^{\dagger}(0.2-61.4)$ & [86] \\
\hline $\begin{array}{l}\text { Jung et al. } \\
\text { (2015) }\end{array}$ & $\mathrm{R}$ & 50 & $65^{\dagger}(30-82)$ & ns & CRC & 79 & GTV: $1.5 \mathrm{ml}^{\dagger}(0.2-34.8)$ & [87] \\
\hline $\begin{array}{l}\text { De Rose et al. } \\
(2016)\end{array}$ & $\mathrm{R}$ & 60 & $70.5^{\dagger}(33-89)$ & $40: 20$ & NSCLC & 90 & $\begin{array}{l}\text { Cumulative volume: } \\
17.5 \mathrm{~cm}^{3} \ddagger(0.9-184)\end{array}$ & [88] \\
\hline Kinj et al. (2016) & $\mathrm{R}$ & 53 & $69^{\dagger}(47-84)$ & $35: 18$ & Re: $32.1 \%$; Co: $67.9 \%$ & 87 & $16 \mathrm{~mm}^{\dagger}(3-70)$ & [33] \\
\hline $\begin{array}{l}\text { Helou et al. } \\
(2016)\end{array}$ & $\mathrm{R}$ & 120 & $67^{\ddagger} \pm 11$ yrs & $58: 62$ & $\begin{array}{l}\text { NSCLC: } 31.3 \% \text {; RCC: } 25.3 \% \text {; B: } \\
21.7 \% ; \text { O: } 21.7 \%\end{array}$ & 184 & $15 \mathrm{~mm}^{\dagger}$ (IQR: 10-25) & [89] \\
\hline $\begin{array}{l}\text { Rieber et al. } \\
(2016)\end{array}$ & $\mathrm{R}$ & 700 & $67^{\dagger}(6.4-99.9)$ & $449: 251$ & $\begin{array}{l}\text { NSCLC: } 30 \% \text {; CRC: } 21.9 \% \text {; S: } \\
7.3 \% \text {; RCC: } 6.9 \% ; \text { B: } 6.2 \% \text {; M: } \\
3.4 \% \text {; Oe: } 2.6 \% ; \text { O: } 21.7 \%\end{array}$ & $\begin{array}{l}1: 42.4 \% ;>1: \\
57.6 \%\end{array}$ & $22 \mathrm{~mm}^{\dagger}(4-94)$ & [4] \\
\hline $\begin{array}{l}\text { Yamashita et al. } \\
\text { (2016) }\end{array}$ & $\mathrm{R}$ & 96 & $72^{\dagger}(25-88)$ & $65: 31$ & $\begin{array}{l}\text { CRC: } 26 \% \text {; NSCLC: } 25 \% \text {; HN: } 8 \% \text {; } \\
\text { U: } 8 \% ; \text { O: } 32 \%\end{array}$ & $\begin{array}{l}1: 79.2 \% ;>1: \\
20.8 \%\end{array}$ & $19 \mathrm{~mm}^{\dagger}(0.6-4.2)$ & [90] \\
\hline $\begin{array}{l}\text { Franceschini } \\
\text { et al. (2017) }\end{array}$ & $\mathrm{R}$ & 200 & $69^{\dagger}(22-90)$ & $123: 77$ & $\begin{array}{l}\text { RCC: } 12 \% ; \text { M: } 4.5 \% \text {; HCC: } 10 \% \text {; } \\
\text { SG: } 3.5 \% ; \text { S: } 20.5 \% ; \text { CRC: } 49.5 \%\end{array}$ & $1: 64 \% ;>1: 36 \%$ & ns & [91] \\
\hline $\begin{array}{l}\text { Ricco et al. } \\
(2017)\end{array}$ & $\mathrm{R}$ & 447 & $69^{\dagger}(18-93)$ & $223: 221$ & $\begin{array}{l}\text { B: } 9.2 \% \text {; CRC: } 25.7 \% \text {; HN: } 11.4 \% \text {; } \\
\text { NSCLC: } 16.6 \% ; \text { RCC: } 8.1 \% ; \text { M: } \\
6.5 \% ; \text { O: } 22.1 \%\end{array}$ & $1^{\dagger}(1-3)$ & $10.58 \mathrm{cc}^{\dagger}(0.1-654.5)$ & [92] \\
\hline Qiu et al. (2018) & $\mathrm{R}$ & 65 & $\begin{array}{l}>60: 60 \% ;<60: \\
40 \%\end{array}$ & $37: 28$ & CRC & $\begin{array}{l}1: 36.9 \% ;>1: \\
63.1 \%\end{array}$ & $\begin{array}{l}<10 \mathrm{~mm}: 27.7 \% ;>1: \\
72.3 \%\end{array}$ & [93] \\
\hline \multicolumn{9}{|c|}{$\begin{array}{l}\text { Values expressed as median/mean (range): } \\
\text { †Median. } \\
\text { †Mean. } \\
\text { B: Breast; Co: Colon; CRC: Colorectal Cancer; GTV: Gross tumor volume; HCC: Hepatocarcinoma; HN: Head \& neck; IQR: Interquartile range; M: Melanoma; ns: Not stated; NSCLC: } \\
\text { Non-small-cell lung cancer; O: Other; Oe: Oesophagus; P: Prospective; Pa: Pancreas; Pat: Patient; Pr: Prostate; R: Retrospective; RCC: Renal Cell Carcinoma; Re: Rectal; S: Sarcoma; } \\
\text { SG: Salivary gland; STS: Soft tissue sarcoma; U: Uterine. }\end{array}$} \\
\hline
\end{tabular}

\section{SBRT}

The description of treatments and length of follow-up is reported in Table 4. A wide range of treatment regimens were found within and across studies, in terms of prescribed doses of radiation and fractions of delivery. Several papers report percentage of isodose delivered at isocenter and PTV and biologically effective doses $\left(\mathrm{BED}_{10}\right)$. All papers reported follow-up period, usually expressed as median and range. Median follow-up time was higher than 2 years in $44.4 \%$ of included articles, however, none of them reported a follow-up time of more than 4 years. 
Table 3. Surgery: treatment and follow-up.

\begin{tabular}{|c|c|c|c|c|c|}
\hline Study (year) & Resection & Approach & RO & Follow-up (mo) & Ref. \\
\hline $\begin{array}{l}\text { Fourquier et al. } \\
\text { (1997) }\end{array}$ & W: 56\%; S: 6\%; L: 34\%; SL: 2\%; P: 10\% & Th: $64 \%$; S: $12 \%$; BTh: $8 \%$; STh: $14 \%$ & ns & $42^{\dagger}(1-200)$ & [12] \\
\hline Robert et al. (1997) & W: $66 \% ;$ S: 5\%; L: 24\%; P: 5\% & Th: $68.8 \%$; TL: $5.1 \%$; St: $17.4 \%$; STh: $8.7 \%$ & $90 \%$ & $38^{\ddagger}(0-161)$ & [44] \\
\hline $\begin{array}{l}\text { Pastorino et al. } \\
\text { (1997) }\end{array}$ & W: 66.7\%; S: 8.6\%; L: 21.3\%; P: 2.5\% & Th: $59.7 \%$; BTh: $11 \%$; St: $27.2 \%$; V: $1.7 \%$ & $88 \%$ & $46^{\dagger}$ & [10] \\
\hline $\begin{array}{l}\text { van der Veen et al. } \\
\text { (1998) }\end{array}$ & W: $94.9 \% ;$ L: $5.1 \%$ & St & $82.1 \%$ & $22 \ddagger(1-132)$ & [13] \\
\hline $\begin{array}{l}\text { Abecasis et al. } \\
\text { (1999) }\end{array}$ & $\begin{array}{l}\text { W: } 55.5 \% ; \text { S: } 2.0 \% ; \text { L: } 32.9 \% ; B: 7.6 \% ; P: \\
2.0 \%\end{array}$ & Th: $91.5 \%$; STh: $6.3 \%$; Cl: $1.1 \%$; V: $1.1 \%$ & ns & $22^{\ddagger}(1-146)$ & [45] \\
\hline $\begin{array}{l}\text { Kolodziejski et al. } \\
\text { (1999) }\end{array}$ & $\begin{array}{l}\text { W: } 33 \% ; \text { S: } 13 \% \text {; L: } 28 \% ; \text { P: } 19.5 \% \text {; ETh: } \\
6.5 \%\end{array}$ & Th: $99 \%$; St: $1 \%$ & ns & ns & [35] \\
\hline Leo et al. (2000) & P: $4.5 \% ;$ L: $26.8 \% ; W / S: 68.7 \%$ & U: $79.4 \% ; B i: 20.6 \%$ & ns & ns & [46] \\
\hline Friedel et al. (2001) & $\begin{array}{l}\text { W: } 61.2 \% ; \text { S: } 12.0 \% \text {; L: } 24.4 \% ; P: 1.3 \% \text {; ns: } \\
1.1 \%\end{array}$ & Th: $75.4 \%$; St: $20.4 \%$; V: $3.6 \%$; ns: $0.6 \%$ & $84 \%$ & $34^{\dagger}(0-240)$ & [47] \\
\hline Piltz et al. (2002) & W: 78.6\%; S: 6\%; L: 12.6\%; B: 3.8\% & ns & ns & ns & [48] \\
\hline $\begin{array}{l}\text { Planchard et al. } \\
\text { (2003) }\end{array}$ & W/S: 74\%; L: 20\%; C: 6\% & Th: $92 \%$; BTh: $8 \%$ & ns & $112.8^{\dagger}(2-264)$ & [49] \\
\hline $\begin{array}{l}\text { Monteiro et al. } \\
\text { (2004) }\end{array}$ & W: $70.4 \%$; L: $28.6 \%$; P: $1 \%$ & ns & ns & $40.8^{\dagger}(3-162)$ & [36] \\
\hline $\begin{array}{l}\text { Vogelsang et al. } \\
\text { (2004) }\end{array}$ & An: $29.8 \% ; W: 70.2 \%$ & ns & $100 \%$ & $33^{\ddagger}(4-116)$ & [50] \\
\hline Shiono et al. (2005) & $\begin{array}{l}\text { W: } 49.5 \% ; \text { S: } 19.5 \% \text {; L: } 28.8 \% ; B: 1.1 \% ; P: \\
1.1 \%\end{array}$ & ns & ns & $32 \ddagger(1-110)$ & [51] \\
\hline Casali et al. (2006) & W: $69 \%$; L: $28 \%$; B: $3 \%$ & ns & Ns & ns & [52] \\
\hline Furak et al. (2006) & W: 54\%; L: 42\%; P: 4\% & U: $90 \% ; \mathrm{Bi}: 10 \%$ & ns & ns & [53] \\
\hline Harting et al. (2006) & ns & U: $58.6 \% ; B i: 41.4 \%$ & $64.7 \%$ & $34.8^{\ddagger}(9-241)$ & [54] \\
\hline Rena et al. (2006) & $\begin{array}{l}\text { W: } 62.5 \% ; \text { S: } 5.6 \% ; \text { L: } 23.8 \% ; C: 4.5 \% ; B: \\
1.6 \% ; \text { P: } 1.9 \%\end{array}$ & Th: $95.5 \%$; St: $1.5 \%$; Cl: $0.5 \%$; STh: $2.5 \%$ & $83.6 \%$ & $25^{\ddagger}(1-218)$ & [55] \\
\hline Melloni et al. (2007) & W: $69.3 \%$; L: $29.5 \%$; P: $1.2 \%$ & Th: $93.2 \%$; St/STh: $6.8 \%$ & ns & $20 \ddagger(4-154)$ & [16] \\
\hline $\begin{array}{l}\text { Veronesi et al. } \\
\text { (2007) }\end{array}$ & W: $40.3 \%$; S: $21.6 \%$; L: $35.2 \%$; P: $2.9 \%$ & Th & ns & $31^{\dagger}(2-82)$ & [56] \\
\hline $\begin{array}{l}\text { Dahabre et al. } \\
\text { (2008) }\end{array}$ & W/S: 45\%; L: 41\%; B 7\%; P: 7\% & ns & $91.2 \%$ & ns & [9] \\
\hline Smith et al. (2008) & W: 78.7\%; L: 18.1\%; P: 3.2\% & ns & $78.7 \%$ & Minimum 60 & [17] \\
\hline $\begin{array}{l}\text { Yoshimoto et al. } \\
(2008)\end{array}$ & W: $11.1 \% ;$ S: $12.2 \%$; L: $75.6 \%$; P: $1.1 \%$ & ns & ns & $79.2^{\ddagger}$ & [37] \\
\hline Winter et al. (2008) & $\begin{array}{l}\text { W: } 77.8 \% ; \text { S: } 12.3 \% ; \text { L: } 6.2 \% ; P: 1.2 \% ; B: \\
2.5 \%\end{array}$ & ns & ns & ns & [57] \\
\hline Lin et al. (2009) & W: $58.7 \% ;$ L: $41.3 \%$ & ns & ns & $37.3^{\ddagger}(12-122)$ & [58] \\
\hline Rama et al. (2009) & W: 78\%; L: 17\%; O: 5\% & Th: $84 \% ;$ BTh: $15 \% ;$ St: $1 \%$ & ns & ns & [59] \\
\hline $\begin{array}{l}\text { Takakura et al. } \\
\text { (2009) }\end{array}$ & W/S: $50 \% ;$ L: $50 \%$ & ns & ns & $30 \ddagger(4-126)$ & {$[60]$} \\
\hline Borasio et al. (2010) & W: $70.8 \% ;$ S: $9.5 \%$; L: $19 \%$; B: $0.7 \%$ & Th: $85.4 \%$; St: $11.7 \%$; BTh: $0.7 \%$; V: $2.2 \%$ & ns & $36.2^{\ddagger}(4.6-126)$ & [61] \\
\hline Riquet et al. 2010 & W/S: 56\%; L: 36.3\%; P: 7.7\% & ns & ns & $46 \ddagger(2-256)$ & [62] \\
\hline $\begin{array}{l}\text { Sardenberg } \\
\text { et al. } 2010\end{array}$ & W/S: $81.8 \% ;$ L: 15.6\%; Bio: $2.6 \%$ & ns & ns & $36.7^{\ddagger}(10-138)$ & [63] \\
\hline Tacconi et al. (2010) & w & HAV & ns & $65^{\dagger}(8-72)$ & [64] \\
\hline $\begin{array}{l}\text { Casiraghi et al. } \\
\text { (2011) }\end{array}$ & $\begin{array}{l}\text { Tu: } 20.8 \% ; \text { W: } 39.2 \% ; \text { S: } 11.8 \% ; \text { L/B: } 27 \% ; P: \\
1.2 \%\end{array}$ & $\begin{array}{l}\text { Th: } 68.8 \% \text {; STh: } 26.3 \% \text {; St: } 2 \% \text {; } \mathrm{HCl}: 0.2 \% \text {; } \\
\text { O: } 0.4 \%\end{array}$ & $85.2 \%$ & $34^{\dagger}$ & [65] \\
\hline \multicolumn{6}{|c|}{$\begin{array}{l}\text { Follow-up values expressed in months as mean/median (range): } \\
\text { †Mean. } \\
\text { ¥Median. } \\
\text { An: Anatomical resection; B: Bilobectomy; Bi: Bilateral; Bio: Biopsy; BTh: Simultaneous bilateral thoracotomy; C: Combined resection; Cl: Clamshell; Eth: Exploratory thora- } \\
\text { cotomy; HAV: Substernal hand-assisted thoracoscopic approach; HCl: Hemiclamshell; IQR: Interquartile range; L: Lobectomy; La: Laser resection; ns: Not stated; O: Other; P: } \\
\text { Pneumonectomy; RO: No residual disease; S: Segmentectomy; SL: sleeve lobectomy; St: Median sternotomy; STh: Staged thoracotomy; Th: Thoracotomy; TL: Thoracolaparo- } \\
\text { tomy: Tu: Tumorectomy; U: Unilateral; V: Video-assisted thoracic surgery; W: Wedge resection. }\end{array}$} \\
\hline
\end{tabular}


Table 3. Surgery: treatment and follow-up (cont.).

\begin{tabular}{|c|c|c|c|c|c|}
\hline Study (year) & Resection & Approach & RO & Follow-up (mo) & Ref. \\
\hline Long et al. (2011) & W: 99.5\%; L: 0.5\% & HAV & ns & $27.4(1-74)$ & [66] \\
\hline $\begin{array}{l}\text { Stephens et al. } \\
\text { (2011) }\end{array}$ & W/S: $85 \%$; L/P: $15 \%$ & ns & ns & $27.0^{\dagger}$ & [67] \\
\hline $\begin{array}{l}\text { Zabaleta et al. } \\
\text { (2011) }\end{array}$ & $\begin{array}{l}\text { W: } 47.5 \% \text {; L: 37.6\%; B: } 1.0 \% \text {; P: } 5.0 \% \text {; C: } \\
5.0 \%\end{array}$ & Th: $91.2 \% ; \mathrm{V}: 6.5 \% ; 0: 2.0 \%$ & ns & $43^{\ddagger}(0-130)$ & [68] \\
\hline $\begin{array}{l}\text { Blackmon et al. } \\
\text { (2012) }\end{array}$ & W: $72.5 \%$; S: $12.2 \%$; L: $15.3 \%$ & V: $16.2 \%$; St/Cl: $8.7 \%$; Th: $73.4 \%$; O: $1.7 \%$ & ns & $37.2^{\ddagger}$ & [18] \\
\hline Chua et al. (2012) & W/S: $51 \% ; 0: 49 \%$ & U: $94 \% ; B i: 6 \%$ & $80 \%$ & $20 \ddagger(0-255)$ & [69] \\
\hline $\begin{array}{l}\text { Goonerante et al. } \\
\text { (2012) }\end{array}$ & W: 54.1\%; L: 42.4\%; C: 7.4\%; P: $2.1 \%$ & ns & ns & $30^{\ddagger}(\mathrm{IQR}: 16-46)$ & [19] \\
\hline $\begin{array}{l}\text { Hirosawa et al. } \\
\text { (2012) }\end{array}$ & W: 42.3\%; S: 38.3\%; L: 32.4\% & U: $87.6 \% ; B i: 22.4 \%$ & $100 \%$ & $74^{\ddagger}(1-188)$ & [70] \\
\hline Mizuno et al. (2012) & W: 84\%; S: 9\%; L: 7\% & V: $59 \%$; Th: $41 \%$ & $92 \%$ & ns & [20] \\
\hline Lo Faso et al. (2013) & W: $64.1 \% ;$ S: $10.4 \%$; L: $25 \% ;$ P: $0.5 \%$ & $\mathrm{v}$ & $97 \%$ & $38^{\dagger}$ & [71] \\
\hline Poletti et al. (2013) & W/S: 51.3\%; Tu: 29.9\%; L/B: 15.6\%; P: 3.2\% & ns & ns & ns & [38] \\
\hline Cho et al. (2014) & W: 77.3\%; S: 5.9\%; L: 15.6\%; P: 0.8\% & Th/St: $76.1 \% ; \mathrm{V}: 23.8 \%$ & ns & $45.5^{\ddagger}(23.0-94.2)$ & [72] \\
\hline Renaud et al. (2014) & W/S: 71\%; L: 22\%; P: 7\% & ns & ns & $33^{\ddagger}$ (IQR: 42.5$)$ & [73] \\
\hline Renaud et al. (2014) & W: 79.5\%; L: $18 \% ;$ P: $2.5 \%$ & Th & ns & ns & [74] \\
\hline $\begin{array}{l}\text { Zampino } \\
\text { et al. (2014) }\end{array}$ & W: $60.3 \%$; S: $13.6 \%$; L: $26.1 \%$ & ns & $89.4 \%$ & $48^{\ddagger}(3.6-172.8)$ & [75] \\
\hline Booth et al. (2015) & L: 40\%; S: 56\%; P: 4\% & ns & ns & ns & [41] \\
\hline Hou et al. (2015) & $\begin{array}{l}\text { VATS: L: } 33.3 \% \text { : W: } 66.7 \% \\
\text { Open: L: } 36.8 \% ; \mathrm{W}: 63.2 \%\end{array}$ & V: $50 \%$; Th: $50 \%$ & $\begin{array}{l}\text { VATS: } 98.2 \% \\
\text { Open: } 100 \%\end{array}$ & $45^{\ddagger}$ & [21] \\
\hline $\begin{array}{l}\text { Seebacher et al. } \\
\text { (2015) }\end{array}$ & W/S/La: 94.5\%; L: 5.5\% & Th & ns & 60 & [76] \\
\hline $\begin{array}{l}\text { Guerrera et al. } \\
\text { (2016) }\end{array}$ & $\begin{array}{l}\text { W: } 68.1 \% ; \text { S: } 8.5 \% ; \text { L: } 20.2 \% ; P: 0.5 \% \text {; C: } \\
2.9 \%\end{array}$ & Th: 80.9\%; St: $18.1 \%$; BTh: $1.0 \%$ & $92 \%$ & $45^{\ddagger}$ (IQR: 30-69) & [77] \\
\hline Filippi et al. (2016) & ns & ns & ns & ns & [30] \\
\hline Franzke et al. (2016) & La: 55.6\%; W: 34.8\%; S: 5.1\%; L: 4.5\% & Th: $74.5 \% ; \mathrm{V}: 25.5 \%$ & $99 \%$ & $23.8^{\ddagger}(2-66)$ & [78] \\
\hline Girelli et al. (2016) & ns & ns & $83.5 \%$ & $52^{\ddagger}$ & [79] \\
\hline Okiror et al. (2016) & ns & V: $73 \%$; Th: $27 \%$ & ns & $31 \ddagger(1-60)$ & [80] \\
\hline $\begin{array}{l}\text { Guerrini et al. } \\
\text { (2016) }\end{array}$ & L: $23 \% ; W / S: 77 \%$ & $\mathrm{v}$ & ns & $40^{\dagger}$ & [39] \\
\hline $\begin{array}{l}\text { Gafencu et al. } \\
\text { (2017) }\end{array}$ & W/La: 85.1\%; S: 4.9\%; L:10.1\% & U: $70.0 \% ; B i: 29.9 \%$ & ns & ns & [22] \\
\hline Kim et al. (2017) & W: 81.4\%; S: $8.5 \% ;$ L: $7.0 \%$; C: $3.1 \%$ & ns & ns & $46.4^{\dagger}(9-111)$ & [23] \\
\hline Shiomi et al. (2017) & W: 76\%; S/L: 24\% & ns & $100 \%$ & 51 & [81] \\
\hline Sun et al. (2017) & W: $66.9 \%$; S: 3.2\%; L: $29.9 \%$ & v & ns & $37^{\ddagger}(2-138)$ & [82] \\
\hline Shiono et al. (2017) & W: $82.3 \% ;$ S: $17.7 \%$ & ns & $100 \%$ & $64.8^{\ddagger}$ & [14] \\
\hline $\begin{array}{l}\text { Lodeweges et al. } \\
\text { (2017) }\end{array}$ & ns & ns & ns & $\begin{array}{l}7.6 \text { years } \\
(5.8-9.8)\end{array}$ & [24] \\
\hline
\end{tabular}

Follow-up values expressed in months as mean/median (range):

$\dagger$ Mean.

$\ddagger$ Median.

An: Anatomical resection; B: Bilobectomy; Bi: Bilateral; Bio: Biopsy; BTh: Simultaneous bilateral thoracotomy; C: Combined resection; Cl: Clamshell; Eth: Exploratory thoracotomy; HAV: Substernal hand-assisted thoracoscopic approach; HCl: Hemiclamshell; IQR: Interquartile range; L: Lobectomy; La: Laser resection; ns: Not stated; O: Other; P: Pneumonectomy; RO: No residual disease; S: Segmentectomy; SL: sleeve lobectomy; St: Median sternotomy; STh: Staged thoracotomy; Th: Thoracotomy; TL: Thoracolaparotomy: Tu: Tumorectomy; U: Unilateral; V: Video-assisted thoracic surgery; W: Wedge resection.

\section{Outcomes \& Complications Surgery}

A description of the prognostic outcomes of surgical treatments and postoperative complications is reported in Table 5. Prognostic outcomes were variously described across studies: a survival analysis has been performed in the vast majority of articles, but intervals for assessing survivors' prevalence ranged widely between short-term ( 1 and 2 years) and long-term lapses (5 and 10 years). Rates of survival at 1, 5 and 10 years ranged between $88-96 \%$, 
Table 4. Stereotactic radiotherapy: treatment and follow-up.

\begin{tabular}{|c|c|c|c|c|c|}
\hline Study (year) & $\mathrm{BED}_{10}$ & Total dose (Gy) & Fractions & Follow-up (mo) & Ref. \\
\hline Hof et al. (2007) & ns & $12-30$ & 1 & $14^{\ddagger}(1.5-82)$ & [6] \\
\hline Ricardi et al. (2011) & ns & $26-45$ & $1-3$ & $20.4^{\ddagger}(3-77.4)$ & [83] \\
\hline Oh et al. (2012) & 100-150 & $50-60$ & $4-5$ & $21^{\ddagger}(3-107)$ & [34] \\
\hline Filippi et al. (2014) & 94-138 & 26 & 1 & $24^{\ddagger}$ & [5] \\
\hline Osti et al. (2013) & $76-120$ & $23-30$ & 1 & $15^{\ddagger}(3-45)$ & [29] \\
\hline Aoki et al. (2015) & $100(85.5-120)$ & $50(45-60)$ & $5(5-9)$ & $31.7^{\ddagger}(4.3-130)$ & [85] \\
\hline Binkley et al. (2015) & $85(50.4-151.2)$ & $25(18-50)$ & ns & $22^{\ddagger}(3-68)$ & [86] \\
\hline Jung et al. (2015) & ns & $40-60$ & $3-4$ & $42.8^{\ddagger}(11.0-104.1)$ & [87] \\
\hline De Rose et al. (2016) & ns & $48-60$ & $3-8$ & $28.1 \mp(5.4-104.5)$ & [88] \\
\hline Kinj et al. (2016) & ns & $50-75$ & $3-5$ & $33^{\ddagger}(4-85)$ & [33] \\
\hline Ricco et al. (2017) & 100 (IQR: 81-136) & 50 (IQR: 48-54) & 3 (IQR: 3-5) & $13^{\ddagger}($ IQR: $6-26)$ & [92] \\
\hline Qiu et al. (2018) & ns & $40-60$ & $5-11$ & $6.4^{\ddagger}$ & [93] \\
\hline
\end{tabular}

$15-76 \%$ and $11-41 \%$, respectively. Recurrence-free survival (RFS) and PFS were reported in a minor proportion of manuscripts (24.6\%). PFS rates at 1 and 5 years were $45-87 \%$ and 5-64\%, respectively. Postoperative mortality was reported in $73.8 \%$ of studies, ranging between 0 and $4.7 \%$. In nearly half of the included studies, a clear mention of the prevalence of complications was not found or could not be derived from presented data. Where reported, overall complication rates ranged between 0 and 23\%. A description or a list of such complications has been found in $59 \%$ of papers.

\section{SBRT}

Outcomes of treatment were reported in an extensive manner in most papers (Table 6). The authors report rates of overall survival (OS) or RFS in almost all articles, with the majority of them (88.9\%) mentioning percentages of LC, reported as rates of LC and progression, local failure-free survival or local response. Intervals for assessing survival and tumor progression were definitely shorter in SBRT papers: only five of the included articles (31.2\%) reported percentages of LC and survival at 5 years. Rates of OS at 1, 2 and 5 years ranged between 74-94.5\%, 31.2-74.6\% and $21.8-58.3 \%$, respectively. PFS at 1 and 2 years was $23.5-72 \%$ and $10-57 \%$, respectively. Mortality rates have been reported in $83.3 \%$ of publications and in $72.2 \%$ of series no patients died as a consequence of ablative treatment. Post-treatment mortality ranged between 0 and $2 \%$. Overall rates of adverse events were described in $27.8 \%$ of papers, whereas a detailed description of them, often with relative percentages or absolute numbers, could be encountered more frequently. Adverse events were almost always reported using the Common Terminology Criteria for Adverse Events [27] and their incidence ranged between 4 and 31\%. In the majority of articles that reported adverse events were Grade 1-Grade 2 toxicity, without the need for further treatments or minor measures only.

\section{Discussion}

Surgical excision of pulmonary oligometastases seems to guarantee improved outcomes in terms of survival, and it is currently the first-choice treatment for this condition [28]. In contrast, SBRT has traditionally been reserved for patients unsuitable for surgical treatment. Nonetheless, recent improvements in technology and more precise protocols are broadening SBRT current indications [29]. It has been proposed that SBRT might induce not only tumor cell death, but also a tumor-specific response of the host immune system, inactivation of remnant micrometastasis 
Systematic Review Londero, Grossi, Morelli et al.

Table 5. Surgery: outcomes and complications.

\begin{tabular}{|c|c|c|c|c|c|c|}
\hline Study (year) & os & PFS & Median survival & Mortality & Morbidity & Ref. \\
\hline $\begin{array}{l}\text { Fourquier et al. } \\
\text { (1997) }\end{array}$ & 5 yrs: $44 \%$ & 5 yrs; $25 \%$ & 25 & $2 \%$ & $6 \%$ & [12] \\
\hline Robert et al. (1997) & 2 yrs: $69 \% ; 5$ yrs: $48 \% ; 10$ yrs: $35 \%$ & ns & ns & $1.8 \%$ & ns & [44] \\
\hline $\begin{array}{l}\text { Pastorino et al. } \\
\text { (1997) }\end{array}$ & 5 yrs: $36 \% ; 10$ yrs: $26 \% ; 15$ yrs: $22 \%$ & ns & 35 & $1 \%$ & ns & [10] \\
\hline $\begin{array}{l}\text { Van der Veen et al. } \\
\text { (1998) }\end{array}$ & 5 yrs: $38 \%$ & ns & ns & $0 \%$ & $15.3 \%$ & [13] \\
\hline $\begin{array}{l}\text { Abecasis et al. } \\
\text { (1999) }\end{array}$ & 5 yrs: $29.2 \%$ & ns & ns & $4.7 \%$ & $21.2 \%$ & [45] \\
\hline $\begin{array}{l}\text { Kolodziejski et al. } \\
\text { (1999) }\end{array}$ & 5 yrs: $58 \% ; 10$ yrs: $38 \%$ & ns & 58 & $2.1 \%$ & $9.6 \%$ & [35] \\
\hline Leo et al. (2000) & 5 yrs: $22 \% ; 10$ yrs: $16 \%$ & ns & 17 & ns & ns & [46] \\
\hline Friedel et al. (2001) & 5 yrs: $35 \% ; 10$ yrs: $20 \% ; 15$ yrs: $18 \%$ & ns & 35 & ns & ns & [47] \\
\hline Piltz et al. (2002) & ns & ns & 61 & $0.95 \%$ & $10.7 \%$ & [48] \\
\hline $\begin{array}{l}\text { Planchard et al. } \\
\text { (2003) }\end{array}$ & 3 yrs: $58 \% ; 5$ yrs: $45 \% ; 10$ yrs: $30 \%$ & $\begin{array}{l}1 \text { yr: } 87 \% ; 3 \text { yrs: } \\
48 \%\end{array}$ & $50.4(2-252)$ & $0.8 \%$ & ns & [49] \\
\hline $\begin{array}{l}\text { Monteiro et al. } \\
\text { (2004) }\end{array}$ & 5 yrs: $47.4 \% ; 10$ yrs: $37.7 \%$ & $\begin{array}{l}5 \text { yrs: } 33.9 \% ; 10 \text { yrs: } \\
19.1 \%\end{array}$ & ns & 0 & $11.5 \%$ & [36] \\
\hline $\begin{array}{l}\text { Vogelsang et al. } \\
\text { (2004) }\end{array}$ & 1 yr: $90 \% ; 3$ yrs: $47 \%$ & ns & ns & $0 \%$ & $3 \%$ & [50] \\
\hline Shiono et al. (2005) & 5 yrs: $61.4 \%$ & ns & ns & $0 \%$ & $\mathrm{~ns}$ & [51] \\
\hline Casali et al. (2006) & 5 yrs: $36 \%$ & 5 yrs: $26 \%$ & ns & ns & ns & [52] \\
\hline Furak et al. (2006) & 5 yrs: $33.6 \%$ & ns & ns & 0 & ns & [53] \\
\hline Harting et al. (2006) & 3 yrs: $46.2 \% ; 5$ yrs: $29 \%$ & ns & ns & ns & $11.8 \%$ & [54] \\
\hline Rena et al. (2006) & 5 yrs: $37 \% ; 10$ yrs: $15.8 \%$ & $\begin{array}{l}5 \text { yrs: } 28 \% ; 10 \text { yrs: } \\
13 \%\end{array}$ & ns & $0.9 \%$ & $7.9 \%$ & [55] \\
\hline $\begin{array}{l}\text { Assouad et al. } \\
(2007)\end{array}$ & 5 yrs: $34.4 \%$ & ns & 35 & 0 & $15.4 \%$ & [15] \\
\hline Melloni et al. (2007) & 3 yrs: $50 \% ; 5$ yrs: $42 \% ; 10$ yrs: $30 \%$ & ns & 37 & ns & $\mathrm{ns}$ & [16] \\
\hline $\begin{array}{l}\text { Veronesi et al. } \\
(2007)\end{array}$ & 5 yrs: $60 \%$ & ns & ns & ns & ns & [56] \\
\hline $\begin{array}{l}\text { Dahabre et al. } \\
\text { (2008) }\end{array}$ & 5 yrs: $32.69 \%$ & ns & 42 & 0 & 0 & [9] \\
\hline Smith et al. (2008) & 5 yrs: $15 \%$ & 5 yrs: $5 \%$ & 16 & $3.7 \%$ & ns & [17] \\
\hline $\begin{array}{l}\text { Yoshimoto et al. } \\
\text { (2008) }\end{array}$ & 5 yrs: $54 \% ; 10$ yrs: $40 \% ; 20$ yrs: $25 \%$ & ns & 75.6 & $1.1 \%$ & ns & [37] \\
\hline Winter et al. (2008) & 5 yrs: $20.9 \%$ & $\mathrm{~ns}$ & 19.4 & $4.4 \%$ & $7.4 \%$ & [57] \\
\hline Lin et al. (2009) & 5 yrs: $43.9 \% ; 10$ yrs: $19.5 \%$ & 5 yrs: $19.5 \%$ & ns & $0 \%$ & $0 \%$ & [58] \\
\hline Rama et al. (2009) & 3 yrs: $61 \% ; 5$ yrs: $48 \% ; 10$ yrs: $11 \%$ & ns & ns & $0 \%$ & $8 \%$ & [59] \\
\hline $\begin{array}{l}\text { Takakura et al. } \\
\text { (2009) }\end{array}$ & 3 yrs: $64.9 \% ; 5$ yrs: $48.2 \%$ & ns & ns & ns & ns & [60] \\
\hline Borasio et al. (2010) & 5 yrs: $55.4 \% ; 10$ yrs: $30.8 \%$ & ns & $28.8(6-99.3)$ & 0 & $13.1 \%$ & [61] \\
\hline Riquet et al. (2010) & 5 yrs: $41 \% ; 10$ yrs: $27 \%$ & ns & 45 & 0 & $14.5 \%$ & [62] \\
\hline $\begin{array}{l}\text { Sardenberg et al. } \\
\text { (2010) }\end{array}$ & 5 yrs: $34.7 \%$ & ns & 36.7 & $0 \%$ & $9.1 \%$ & [63] \\
\hline Tacconi et al. (2010) & 3 yrs: $57.9 \% ; 5$ yrs: $38.4 \%$ & $\begin{array}{l}3 \text { yrs: } 53.5 \% ; 5 \text { yrs: } \\
40.9 \%\end{array}$ & ns & $0 \%$ & $2.3 \%$ & [64] \\
\hline $\begin{array}{l}\text { Casiraghi et al. } \\
\text { (2011) }\end{array}$ & 2 yrs: $74 \% ; 5$ yrs: $46 \%$ & ns & ns & 0 & $13.8 \%$ & [65] \\
\hline Long et al. (2011) & 3 yrs: $59.8 \% ; 5$ yrs: $47.2 \%$ & ns & 40 & 0 & $3.6 \%$ & [66] \\
\hline $\begin{array}{l}\text { Stephens et al. } \\
\text { (2011) }\end{array}$ & ns & ns & 24.1 & ns & ns & [67] \\
\hline $\begin{array}{l}\text { Zabaleta et al. } \\
\text { (2011) }\end{array}$ & 3 yrs: $70.2 \% ; 5$ yrs: $54.3 \%$ & ns & $72(0-129)$ & $2 \%$ & $8.3 \%$ & [68] \\
\hline
\end{tabular}


Table 5. Surgery: outcomes and complications (cont.).

\begin{tabular}{|c|c|c|c|c|c|c|}
\hline Study (year) & os & PFS & Median survival & Mortality & Morbidity & Ref. \\
\hline $\begin{array}{l}\text { Blackmon et al. } \\
\text { (2012) }\end{array}$ & 5 yrs: $55.4 \%$ & ns & 70.1 & 0 & $1.7 \%$ & [18] \\
\hline Chua et al. (2012) & 3 yrs: $41 \% ; 5$ yrs: $34 \%$ & $\begin{array}{l}1 \text { yr: } 45 \% ; 3 \text { yrs: } \\
25 \%\end{array}$ & 23 & $1 \%$ & ns & [69] \\
\hline $\begin{array}{l}\text { Goonerante et al. } \\
\text { (2012) }\end{array}$ & 5 yrs: $40 \%$ & ns & ns & $0 \%$ & $14 \%$ & [19] \\
\hline $\begin{array}{l}\text { Hirosawa et al. } \\
\text { (2012) }\end{array}$ & 2 yrs: $76.6 \% ; 5$ yrs: $46.7 \%$ & ns & ns & 0 & ns & [70] \\
\hline Mizuno et al. (2012) & ns & ns & 50 & ns & $7.6 \%$ & [20] \\
\hline Lo Faso et al. (2013) & ns & ns & ns & 0 & Major $2.4 \%$ & [71] \\
\hline Poletti et al. (2013) & $\begin{array}{l}1 \text { yr: } 96 \% ; 3 \text { yrs: } 77 \% ; 5 \text { yrs: } 56 \% ; 10 \text { yrs: } \\
39 \%\end{array}$ & ns & ns & $1.9 \%$ & $22.1 \%$ & [38] \\
\hline Cho et al. (2014) & 5 yrs: $55.6 \%$ & 5 yrs: $64.1 \%$ & ns & ns & ns & [72] \\
\hline Renaud et al. (2014) & ns & ns & ns & $2 \%$ & ns & [73] \\
\hline Renaud et al. (2014) & $\begin{array}{l}1 \text { yr: } 88 \% ; 2 \text { yrs: } 72 \% ; 3 \text { yrs: } 66 \% ; 5 \text { yrs: } \\
58 \%\end{array}$ & ns & 94 & ns & ns & [74] \\
\hline $\begin{array}{l}\text { Zampino et al. } \\
\text { (2014) }\end{array}$ & 5 yrs: $43 \%$ & ns & 50.4 & $0 \%$ & ns & [75] \\
\hline Booth et al. (2015) & 5 yrs: $40 \% ; 10$ yrs: $27 \%$ & ns & ns & $0.9 \%$ & ns & [41] \\
\hline Hou et al. (2015) & 5 yrs: VATS: $50 \%$; Open: $46 \%$ & ns & ns & $0 \%$ & $\begin{array}{l}\text { VATS: } 19 \% \text {; open: } \\
23 \%\end{array}$ & [21] \\
\hline $\begin{array}{l}\text { Seebacher et al. } \\
\text { (2015) }\end{array}$ & 5 yrs: $27.9 \%$ & ns & ns & $0.3 \%$ & $5.7 \%$ & [76] \\
\hline $\begin{array}{l}\text { Guerrera et al. } \\
\text { (2016) }\end{array}$ & 2 yrs: $80 \% ; 5$ yrs: $53 \%$ & $\begin{array}{l}2 \text { yrs: } 54 \% ; 5 \text { yrs: } \\
33 \%\end{array}$ & ns & $0.5 \%$ & $2 \%$ & [77] \\
\hline Filippi et al. (2016) & 1 yr: $96 \% ; 2$ yrs: $82 \%$ & ns & ns & $1(0.7 \%)$ & $0 \%$ & [30] \\
\hline Franzke et al. (2016) & 1 yr: $88.2 \% ; 3$ yrs: $71.4 \% ; 5$ yrs: $69.3 \%$ & ns & $51.0(46.9-55.1)$ & 0 & $11.5 \%$ & [78] \\
\hline Girelli et al. (2016) & 5 yrs: $66.8 \% ; 10$ yrs: $40.5 \%$ & ns & 90.9 & ns & ns & [79] \\
\hline Okiror et al. (2016) & ns & ns & $25.5(1-60)$ & $0 \%$ & ns & [80] \\
\hline $\begin{array}{l}\text { Guerrini et al. } \\
\text { (2016) }\end{array}$ & $\begin{array}{l}1 \text { yr: } 88.2 \% ; 3 \text { yrs: } 71.4 \% ; 5 \text { yrs: } 60.6 \% \text {; } \\
10 \text { yrs: } 41.3 \%\end{array}$ & ns & ns & $0 \%$ & ns & [39] \\
\hline $\begin{array}{l}\text { Gafencu et al. } \\
\text { (2017) }\end{array}$ & ns & ns & ns & 0 & $2.75 \%$ & [22] \\
\hline Kim et al. (2017) & 5 yrs: $62.9 \%$ & 3 yrs: $50.7 \%$ & ns & ns & ns & [23] \\
\hline Shiomi et al. (2017) & 5 yrs: $76 \%$ & 5 yrs: $41 \%$ & ns & ns & ns & [81] \\
\hline Sun et al. (2017) & 5 yrs: $71.3 \%$ & ns & ns & ns & ns & [82] \\
\hline Shiono et al. (2017) & 5 yrs: $70.5 \%$ & 5 yrs: $38.2 \%$ & ns & $0 \%$ & $6.5 \%$ & [14] \\
\hline $\begin{array}{l}\text { Lodeweges et al. } \\
\text { (2017) }\end{array}$ & 5 yrs: $41 \%$ & $1 \mathrm{yr}: 56 \%$ & ns & ns & ns & [24] \\
\hline
\end{tabular}

and improved control of disease, in what is known as 'abscopal effect' [30]. Consequently, some authors evaluated SBRT as an effective and less invasive alternative to surgery for patients with lung oligometastases [31].

The aim of this study was to report the current evidence on these two approaches for treatment of pulmonary metastases and compare them in terms of effectiveness and safety.

The main findings of our study can be summarized as follows:

- We failed to demonstrate any substantial difference between surgery and SBRT in terms of short-term survival results.

- Data on long-term outcomes suggest an advantage for surgery in terms of survival.

- The incidence of adverse events was overall similar in the reported articles, although in the radiotherapy articles an overestimation of adverse events has been observed. 
Table 6. Stereotactic radiotherapy: outcomes and complications.

\begin{tabular}{|c|c|c|c|c|c|c|}
\hline Study (year) & os & PFS & LC & Mortality & Morbidity & Ref. \\
\hline Hof et al. (2007) & 1 yr: $78.4 \% ; 2$ yrs: $65.1 \% ; 3$ yrs: $47.8 \%$ & ns & $\begin{array}{l}1 \text { yr: } 88.6 \% ; 2 \text { yrs: } 73.7 \% ; \\
3 \text { yrs: } 63.1 \%\end{array}$ & $0 \%$ & ns & [6] \\
\hline $\begin{array}{l}\text { Ricardi et al. } \\
\text { (2011) }\end{array}$ & 2 yrs: $66.5 \%$ & 2 yrs: $32.4 \%$ & ns & $0 \%$ & $4.92 \%$ & [83] \\
\hline $\begin{array}{l}\text { Zhang et al. } \\
\text { (2011) }\end{array}$ & 1 yr: $78.9 \% ; 3$ yrs: $40.8 \% ; 5$ yrs: $25.2 \%$ & ns & $\begin{array}{l}1 \text { yr: } 96.6 \% ; 3 \text { yrs: } 89.4 \% \\
5 \text { yrs: } 89.4 \%\end{array}$ & $0 \%$ & ns & [25] \\
\hline Oh et al. (2012) & 2 yrs: $59.7 \% ; 5$ yrs: $56.2 \%$ & ns & ns & $2 \%$ & ns & [34] \\
\hline $\begin{array}{l}\text { Filippi et al. } \\
\text { (2014) }\end{array}$ & 1 yr: $85.1 \% ; 2$ yrs: $70.5 \%$ & $\begin{array}{l}1 \text { yr: } 72 \% ; 2 \text { yrs: } \\
55.4 \%\end{array}$ & 1 yr: $93.4 \% ; 2$ yrs: $88.1 \%$ & $0 \%$ & ns & [5] \\
\hline Osti et al. (2013) & 1 yr: $76.4 \% ; 2$ yrs: $31.2 \%$ & $\begin{array}{l}1 \text { yr: } 53.9 \% ; 2 \text { yrs: } \\
22 \%\end{array}$ & 1 yr: $89.1 \% ; 2$ yrs: $82.1 \%$ & $0 \%$ & ns & [29] \\
\hline Siva et al. (2015) & 1 yr: $93 \% ; 2$ yrs: $71 \%$ & ns & ns & $0 \%$ & $31 \%$ & [84] \\
\hline $\begin{array}{l}\text { Aoki et al. } \\
\text { (2015) }\end{array}$ & 3 yrs: $76 \%$ & 3 yrs: $53.7 \%$ & 3 yrs: $90 \%$ & $0 \%$ & $5 \%$ & [85] \\
\hline $\begin{array}{l}\text { Binkley et al. } \\
\text { (2015) }\end{array}$ & 1 yr: $93.7 \% ; 2$ yrs: $74.6 \%$ & ns & 1 yr: $91.3 \% ; 2$ yr: $83.8 \%$ & $0 \%$ & ns & [86] \\
\hline $\begin{array}{l}\text { Jung et al. } \\
\text { (2015) }\end{array}$ & 3 yrs: $64 \%$ & 3 yrs: $24 \%$ & 1 yr: $88.7 \% ; 3$ yrs: $70.6 \%$ & $0 \%$ & $4 \%$ & [87] \\
\hline $\begin{array}{l}\text { De Rose et al. } \\
\text { (2016) }\end{array}$ & $\begin{array}{l}1 \text { yr: } 94.5 \% ; 2 \text { yrs: } 74.6 \% ; 3 \text { yrs: } 64.3 \% \text {; } \\
5 \text { yrs: } 22.1 \%\end{array}$ & ns & ns & $0 \%$ & ns & [88] \\
\hline Kinj et al. (2016) & 1 yr: $83.8 \% ; 2$ yrs: $69.3 \% ; 5$ yrs: $58.3 \%$ & $\begin{array}{l}1 \text { yr: } 29.2 \% ; 2 \text { yrs: } \\
14.6 \%\end{array}$ & 1 yr: $79.8 \% ; 2$ yrs: $78.2 \%$ & $0 \%$ & ns & [33] \\
\hline $\begin{array}{l}\text { Helou et al. } \\
\text { (2016) }\end{array}$ & ns & ns & 1 yr: $95.6 \% ; 2$ yrs: $84.8 \%$ & ns & $8.3 \%$ & [89] \\
\hline $\begin{array}{l}\text { Rieber et al. } \\
\text { (2016) }\end{array}$ & 1 yr: $75.1 \% ; 2$ yrs: $54.4 \%$ & ns & 1 yr: $90.9 \% ; 2$ yrs: $81.2 \%$ & $0.2 \%$ & ns & [4] \\
\hline $\begin{array}{l}\text { Yamashita et al. } \\
\text { (2016) }\end{array}$ & 3 yrs: $53.2 \%$ & 3 yrs: $32.2 \%$ & 3 yrs: $74.2 \%$ & $0 \%$ & ns & [90] \\
\hline $\begin{array}{l}\text { Franceschini } \\
\text { et al. (2017) }\end{array}$ & ns & $\begin{array}{l}1 \text { yr: } 84 \% ; 2 \text { yrs: } \\
57.7 \% ; 3 \text { yrs: } 47 \%\end{array}$ & $\begin{array}{l}1 \text { yr: } 91 \% ; 2 \text { yrs: } 84.9 \% ; 3 \text { yrs: } \\
82 \%\end{array}$ & $0 \%$ & ns & [91] \\
\hline $\begin{array}{l}\text { Ricco et al. } \\
\text { (2017) }\end{array}$ & 1 yr: $74.1 \% ; 3$ yrs: $33.3 \% ; 5$ yrs: $21.8 \%$ & ns & $\begin{array}{l}1 \text { yr: } 80.4 \% ; 3 \text { yrs: } 58.9 \% \\
5 \text { yrs: } 46.2 \%\end{array}$ & ns & ns & [92] \\
\hline Qiu et al. (2018) & 1 yr: $77.8 \% ; 2$ yrs: $42.8 \%$ & $\begin{array}{l}1 \text { yr: } 23.5 \% ; 2 \text { yrs: } \\
10.1 \%\end{array}$ & 1 yr: $56.6 \% ; 2$ yrs: $30.9 \%$ & ns & ns & [93] \\
\hline
\end{tabular}

In the selected studies, OS at 1 year was $71-96 \%$ in surgical series and $74-94.5 \%$ after radiation therapy. A recent retrospective study comparing the two treatments for metastases from colorectal cancer demonstrated similar rates of survival in the short term, without significant differences in the two groups [32]. These figures are expression of a good outcome after resection/irradiation in the short term, but it is not clear whether this is a consequence of local ablative treatments or part of the natural history of the disease [33]. Patients with an oligometastatic state are a particular subset of subjects with favorable prognostic factors [33] and therefore, short-term survival is probably not the best parameter to establish the comparative effectiveness of these two treatments. A more appropriate indicator of therapeutic efficacy in the short term might be the PFS. In the present review, PFS was slightly higher in the surgical studies. In a recent retrospective study comparing surgery and SBRT for lung metastases, Filippi et al. demonstrated a worse outcome in terms of PFS and local progression among patients in the SBRT group [32]. Since in the context of metastatic disease the main goal of treatment is not cure but delaying progression and prolonging survival [34], the most important point is performing a procedure that eliminates any possible source of further metastatic spread. The presence of residual tumor has been demonstrated to be the main negative prognostic factor in many large surgical studies, some of them published in the late 90s [11]. While surgical excision with evaluation of resection margins allows an immediate feedback of completeness of resection, the efficacy of SBRT is defined with the concept of LC and it can only be assessed at follow-up investigations. The primary goal of SBRT for the treatment of oligometastases is to achieve an excellent rate of LC [31]. Although the R parameter refers to the degree of completeness of surgical resection and LC is a late measure of effectiveness of SBRT treatment, there is an evident analogy between the two factors in terms of potential eradication of disease. In the reported studies, slightly better 
rates of complete resection in the surgical series have been described when compared with LC at 1 year. This may have a significant impact on recurrence and PFS and therefore, on long-term prognosis.

Only a minority of reports on SBRT have a follow-up time long enough to describe long-term outcomes data, but from the included studies OS at 5 years tends to be higher in the surgical series. However, these results should be interpreted with caution, since SBRT is currently still reserved for patients unfit or unwilling to undergo a surgical treatment and this might have a considerable impact on the baseline conditions and survival rates of SBRT series populations. In SBRT studies the best outcomes seem to be reached with high doses of radiation: in the study from Kinj et al. [35]. 5-years OS reached 58.3\% and in the series of Oh et al., this was 56\% [36]. This confirms that high doses of radiation conveyed to the target nodule, expressed as biological effective dose $\left(\mathrm{BED}_{10}\right)$, result in better ablative power and control of disease [34]. However, the effectiveness of SBRT seems to depend not only on the irradiation protocols but also on the pattern of development of the oligometastatic state: in a recent large Japanese nationwide series, patients with a metachronous oligometastatic state demonstrated higher OS rates compared with those with synchronous oligometastasis [10]. Nonetheless, increasing attention is being paid toward the evolution of the pattern of disease in response to systemic therapy or previous local ablative treatments and future investigations will likely address the role of repeat-oligometastasis, the differences between a genuine or a chemotherapy-induced oligometastatic state and their impact on the prognosis of patients [37]. Moreover, the rapid evolution of systemic treatments and the introduction of molecular-targeted therapies might change the role of local aggressive treatments in this setting [38].

In the surgical series from Smith et al., the authors report a low survival rate of $15 \%$ at 5 years and in the same study, complete resection resulted the most relevant factor affecting survival [18]. In this study the prevalence of R0 resections was low (78\%), confirming the importance of a complete eradication of tumor deposits during surgical treatment. Apart from this exceptions, 5-years OS is usually greater in surgical studies and can be as high as $76 \%$. Moreover, several surgical series describe rates of survival at 10 years reaching $40 \%$ [39-43], suggesting that a stabilization or eradication of disease can be obtained in some cases.

Complications and adverse events were reported with similar percentages between surgical and SBRT series, but this is probably the result of a reporting bias: there is an overestimation of adverse events after SBRT, due to reporting of complications that do not require any further therapeutic measure.

There are consistent differences between surgical excision of metastases and other ablative techniques: excision of lung nodules allows availability of tissue for confirmation of the metastatic nature of nodules, assessment of resection margin for complete resection and pathological study for existing or future targeted therapies [44].

SBRT is an ablative technique that does not allow tissue harvesting for confirmation of diagnosis. Thus, one may wonder whether all sites of irradiation effectively correspond to metastatic deposits. Indication for irradiation is usually given at multidisciplinary tumor boards, based on patients' past medical history, radiological features of the nodules and dimensional evolution. Nevertheless, even with high levels of suspicion, some benign nodules may mimic tumor features in radiological examinations. Indeed, several surgical investigations report how some excised nodules did not confirm their metastatic nature at histopathological analysis [45,46]. Therefore, including patients with benign nodules treated with SBRT might overestimate the survival benefits in the whole population.

Another element of concern could be that treating patients with SBRT means not having any information regarding the R0 status after treatment or possible nodal involvement. Moreover, a suboptimal sensitivity of preoperative imaging techniques (CT, CT-PET) has been demonstrated [47] and unexpected further lung nodules at thoracotomy are not an uncommon finding $[46,47]$. For SBRT, this might implicate that radiation is not delivered on a potential source of further tumor dissemination.

Finally, our investigation demonstrated that surgical series provide information based on larger populations and with longer follow-up times. This makes, in our opinion, results coming from surgical reports more reliable. As long as greater experience will mature in the use of SBRT and more solid evidence will emerge from large series, we still recommend surgery as the main therapeutic option for oligometastatic disease of the lung. A prospective randomized protocol comparing the two techniques is required to confirm our conclusions.

\section{Limitations}

There are several limitations in this study. The low number of articles directly comparing the two therapeutic options lead the authors to include series dealing with one of the two treatments only. Nonetheless, most of the articles reported incomplete information on outcomes and baseline conditions of patients and this may have reduced the reliability of results. The net prevalence of retrospective studies and the presence of bias within and across them 
may have limited the accuracy of reported evidence. Including studies with metastases from several primary tumors may have limited the significance of the outcome results, but this was determined by the fact that most SBRT series deal with metastases arising from multiple histology. SBRT is usually reserved to patients excluded from surgical indication due to anatomical considerations or general conditions: this may have an impact on prognostic outcomes. SBRT articles described adverse events in a more accurate manner, reporting even low-grade events where there was no need for any intervention: this undoubtedly overestimated the overall reported incidence of complications compared with surgical series. Nonetheless many surgical articles did not report the $\mathrm{R}$ category, which is an aspect of main importance when dealing with a therapeutic resection for a neoplastic state.

\section{Conclusion}

Surgical resection of pulmonary oligometastases is a safe and effective practice and allows a good control of disease and prolongation of life, in cases where a complete resection can be achieved. SBRT is an attractive option with lower invasiveness and side effects, but long-term follow-up data are still limited. Nonetheless, the heterogeneity of therapy protocols in SBRT and the intrinsic differences between the two treatments do not allow to draw a conclusion on the superiority of one option over the other.

\section{Future perspective}

The recent technological evolution led to a better understanding of the tumors' behavior and the introduction of new molecules changed the view of metastatic disease from being a condition of negative outcome to a stage where the tumor can be stabilized or eradicated. This draws interesting perspectives:

- What will be the role of local treatments in the context of an 'induced oligometastatic state' after systemic therapy regimens administered on patients with an overt polimetastatic disease?

- In this setting, it would be of great interest to assess the new roles of the different ablative techniques during the evolution of disease;

- Nonetheless, a Phase III trial with long follow-up times and strict inclusion criteria would be recommended to compare the effectiveness of the different aggressive local treatments in the context of oligometastatic disease.

Executive summary

- An oligometastatic state is a condition with a limited number of metastases, where local aggressive treatments, such as surgical resection or stereotactic radiotherapy (SBRT), may play an important therapeutic role.

- Aim of the study was to define which is the best local treatment for patients with an oligometastatic disease.

- The study was conducted according to the PRISMA guidelines for systematic review and meta-analysis and literature search was performed by defining a searching strategy using the PICOS method.

- Only articles in English language, with populations of more than 50 patients and where the treatment was performed with curative intent were included.

- Sixty-one surgical studies and 18 articles on SBRT were finally selected.

- Overall survival is comparable in the short term but tends to be higher in the long term, although there is a scarcity of long-term results for SBRT.

- Progression-free survival at 1 and 2 years tends to be higher in surgical studies.

- Mortality is comparable between surgery and SBRT, while the incidence of significant adverse events is higher in patients undergoing surgical resection.

- The intrinsic differences between the two techniques and the heterogeneity of reporting of the included articles do not consent to define which is the best treatment.

- Future investigations should include a prospective trial to compare the two modalities of treatment and define the role of the different ablative techniques in the era of new chemotherapy regimens.

\section{Author contributions}

F Londero contributed to the study conception and design, articles research and selection, data collection, manuscript draft and revision. W Grossi was responsible for the study design, articles research and selection, data collection, manuscript draft. A Morelli is also responsible for the study design, draft and revision of manuscript. O Parise performed data collection and organization, manuscript draft. G Masullo contributed to the study design, manuscript draft. C Tetta prepared the manuscript draft and revision. $U$ Livi was responsible for the articles eligibility assessment, assessment of risk of bias, manuscript revision. G Maessen performed articles eligibility assessment, assessment of risk of bias, manuscript revision. S Gelsomino was responsible for the study conception and design, review process supervision, manuscript draft and revision. 
Financial \& competing interests disclosure

The authors have no relevant affiliations or financial involvement with any organization or entity with a financial interest in or financial conflict with the subject matter or materials discussed in the manuscript. This includes employment, consultancies, honoraria, stock ownership or options, expert testimony, grants or patents received or pending, or royalties.

No writing assistance was utilized in the production of this manuscript.

\section{Open access}

This work is licensed under the Creative Commons Attribution 4.0 License. To view a copy of this license, visit http://creativecommons.org/licenses/by/4.0/

\section{References}

Papers of special note have been highlighted as: $\bullet$ of interest; $\bullet \bullet$ of considerable interest

1. Gloeckler Ries LA, Reichman ME, Lewis DR, Hankey BF, Edwards BK. Cancer survival and incidence from the Surveillance, Epidemiology, and End Results (SEER) program. Oncologist 8(6), 541-552 (2003).

2. Sauter ER, Bolton JS, Willis GW, Farr GH, Sardi A. Improved survival after pulmonary resection of metastatic colorectal carcinoma. J. Surg. Oncol. 43(3), 135-138 (1990).

3. Timmerman RD, Bizekis CS, Pass HI et al. Local surgical, ablative, and radiation treatment of metastases. CA Cancer J. Clin. 59(3), 145-170 (2009).

4. Rieber J, Streblow J, Uhlmann L et al. Stereotactic body radiotherapy (SBRT) for medically inoperable lung metastases-a pooled analysis of the German working group "stereotactic radiotherapy". Lung Cancer 97, 51-58 (2016).

5. Filippi AR, Badellino S, Guarneri A et al. Outcomes of single fraction stereotactic ablative radiotherapy for lung metastases. Technol. Cancer Res. Treat 13(1), 37-45 (2014).

6. Hof H, Hoess A, Oetzel D, Debus J, Herfarth K. Stereotactic single-dose radiotherapy of lung metastases. Strablenther Onkol. 183(12), 673-678 (2007).

7. Moher D, Liberati A, Tetzlaff J, Altman DG. Preferred reporting items for systematic reviews and meta-analyses: the PRISMA statement. BMJ (Clin. Rese. Ed.) 339, b2535 (2009).

8. O'Connor DGS, Higgins JPT. Chapter 5: defining the review question and developing criteria for including studies. In: Cochrane Handbook for Systematic Reviews and Interventions Version 5.1.0. (2008).

9. Dahabre J, Vasilaki M, Stathopoulos GP et al. Surgical management in lung metastases from colorectal cancer. Anticancer Res. 27(6c), 4387-4390 (2007).

10. Niibe $\mathrm{Y}$, Yamamoto $\mathrm{T}$, Onishi $\mathrm{H}$ et al. Pulmonary oligometastases treated by stereotactic body radiation therapy: a nationwide survey of 1,378 patients. Anticancer Res. 40(1), 393-399 (2020).

-• The largest series on lung oligometastases treated with stereotactic radiotherapy.

11. Pastorino U, Buyse M, Friedel G et al. Long-term results of lung metastasectomy: prognostic analyses based on 5206 cases. J. Thorac. Cardiovasc. Surg. 113(1), 37-49 (1997).

-• The first large international series of patients submitted to pulmonary metastasectomy.

12. Verazin GT, Warneke JA, Driscoll DL, Karakousis C, Petrelli NJ, Takita H. Resection of lung metastases from soft-tissue sarcomas. A multivariate analysis. Arch. Surg. 127(12), 1407-1411 (1992).

13. Fourquier P, Regnard JF, Rea S, Levi JF, Levasseur P. Lung metastases of renal cell carcinoma: results of surgical resection. Eur. J. Cardiothorac. Surg. 11(1), 17-21 (1997).

14. van der Veen AH, van Geel AN, Hop WC, Wiggers T. Median sternotomy: the preferred incision for resection of lung metastases. Eur. J. Surg. 164(7), 507-512 (1998).

15. Shiono S, Ishii G, Nagai K et al. Histopathologic prognostic factors in resected colorectal lung metastases. Ann. Thorac. Surg. 79(1), 278-282 (2005).

16. Assouad J, Petkova B, Berna P, Dujon A, Foucault C, Riquet M. Renal cell carcinoma lung metastases surgery: pathologic findings and prognostic factors. Ann. Thorac. Surg. 84(4), 1114-1120 (2007).

17. Melloni G, Doglioni C, Bandiera A et al. Prognostic factors and analysis of microsatellite instability in resected pulmonary metastases from colorectal carcinoma. Ann. Thorac. Surg. 81(6), 2008-2013 (2006).

18. Smith R, Pak Y, Kraybill W, Kane JM 3rd. Factors associated with actual long-term survival following soft tissue sarcoma pulmonary metastasectomy. Eur. J. Surg. Oncol. 35(4), 356-361 (2009).

19. Blackmon SH, Stephens EH, Correa AM et al. Predictors of recurrent pulmonary metastases and survival after pulmonary metastasectomy for colorectal cancer. Ann. Thorac. Surg. 94(6), 1802-1809 (2012).

20. Goonerante D, Gray C, Lim M et al. Survival outcome in New Zealand after resection of colorectal cancer lung metastases. ANZ. J. Surg 83(12), 959-962 (2013). 
21. Mizuno T, Taniguchi T, Ishikawa Y et al. Pulmonary metastasectomy for osteogenic and soft tissue sarcoma: who really benefits from surgical treatment? Eur. J. Cardiothorac. Surg. 43(4), 795-799 (2013).

22. Hou Z, Zhang H, Gui L, Wang W, Zhao S. Video-assisted thoracoscopic surgery versus open resection of lung metastases from colorectal cancer. Int. J. Clin. Exp. Med. 8(8), 13571-13577 (2015).

23. Gafencu DA, Welter S, Cheufou DH et al. Pulmonary metastasectomy for sarcoma-Essen experience. J. Thorac. Dis 9(Suppl. 12), S1278-S1281 (2017).

24. Kim JY, Park IJ, Kim HR et al. Post-pulmonary metastasectomy prognosis after curative resection for colorectal cancer. Oncotarget 8(22), 36566-36577 (2017).

25. Lodeweges JE, Klinkenberg TJ, Ubbels JF, Groen HJM, Langendijk JA, Widder J. Long-term outcome of surgery or stereotactic radiotherapy for lung oligometastases. J. Thorac. Oncol 12(9), 1442-1445 (2017).

26. Zhang Y, Xiao JP, Zhang HZ et al. Stereotactic body radiation therapy favors long-term overall survival in patients with lung metastases: five-year experience of a single-institution. Chin. Med. J. 124(24), 4132-4137 (2011).

27. Trotti A, Colevas AD, Setser A et al. CTCAE v3.0: development of a comprehensive grading system for the adverse effects of cancer treatment. Semin. Radiat. Oncol. 13(3), 176-181 (2003).

28. Navarria P, Ascolese AM, Cozzi L et al. Stereotactic body radiation therapy for lung metastases from soft tissue sarcoma. Eur. J. Cancer 51(5), 668-674 (2015).

29. DeLaney TF, Trofimov AV, Engelsman M, Suit HD. Advanced-technology radiation therapy in the management of bone and soft tissue sarcomas. Cancer Control. 12(1), 27-35 (2005).

30. Niibe Y, Chang JY. Novel insights of oligometastases and oligo-recurrence and review of the literature. Pulm. Med. 2012, 261096-261096 (2012).

31. Osti MF, Carnevale A, Valeriani M et al. Clinical outcomes of single dose stereotactic radiotherapy for lung metastases. Clin. Lung Cancer 14(6), 699-703 (2013).

32. Filippi AR, Guerrera F, Badellino $S$ et al. Exploratory analysis on overall survival after either surgery or stereotactic radiotherapy for lung oligometastases from colorectal cancer. Clin. Oncol. 28(8), 505-512 (2016).

33. Treasure T, Milosevic M, Fiorentino F, Macbeth F. Pulmonary metastasectomy: what is the practice and where is the evidence for effectiveness? Thorax 69(10), 946-949 (2014).

- Interesting paper analyzing the controversies of the practice of pulmonary metastasesctomy.

34. Tree AC, Khoo VS, Eeles RA et al. Stereotactic body radiotherapy for oligometastases. Lancet Oncol. 14(1), e28-e37 (2013).

35. Kinj R, Bondiau PY, Francois E et al. Radiosensitivity of colon and rectal lung oligometastasis treated with stereotactic ablative radiotherapy. Clin. Colorectal Cancer 16(3), e211-e220 (2017).

36. Oh D, Ahn YC, Seo JM et al. Potentially curative stereotactic body radiation therapy (SBRT) for single or oligometastasis to the lung. Acta. Oncol. 51(5), 596-602 (2012).

37. Guckenberger M, Lievens $\mathrm{Y}$, Bouma $\mathrm{AB}$ et al. Characterisation and classification of oligometastatic disease: a European Society for Radiotherapy and Oncology and European Organisation for Research and Treatment of Cancer consensus recommendation. Lancet Oncol. 21(1), e18-e28 (2020).

-• Defines the various subgroups of oligometastatic disease with important prognostic impact.

38. Niibe Y, Hayakawa K. Oligometastases and oligo-recurrence: the new era of cancer therapy. Jpn. J. Clin. Oncol. 40(2), 107-111 (2010).

39. Koodziejski L, Goralczyk J, Dyczek S, Duda K, Nabiaek T. The role of surgery in lung metastases. Eur. J. Surg. Oncol. 25(4), 410-417 (1999).

40. Monteiro A, Arce N, Bernardo J, Eugenio L, Antunes MJ. Surgical resection of lung metastases from epithelial tumors. Ann. Thorac. Surg. 77(2), 431-437 (2004)

41. Yoshimoto M, Tada K, Nishimura $\mathrm{S}$ et al. Favourable long-term results after surgical removal of lung metastases of breast cancer. Breast Cancer Res. Treat. 110(3), 485-491 (2008).

42. Poletti GB, Toro IF, Alves TF, Miranda EC, Seabra JC, Mussi RK. Descriptive analysis of and overall survival after surgical treatment of lung metastases. J. Bras. Pneumol. 39(6), 650-658 (2013).

43. Guerrini GP, Lo Faso F, Vagliasindi A et al. The role of minimally invasive surgery in the treatment of lung metastases. J. Invest. Surg. 30(2), 110-115 (2017).

44. Treasure T. Surgery and ablative techniques for lung metastases in the Pulmonary Metastasectomy in Colorectal Cancer (PulMiCC) trial: is there equivalence? J. Thorac. Dis. 8(Suppl. 9), S649-S651 (2016).

- Analyzes the differences between surgical resection and irradiation of lung oligometastases.

45. Booth CM, Nanji S, Wei X, Mackillop WJ. Outcomes of resected colorectal cancer lung metastases in routine clinical practice: a population-based study. Ann. Surg. Oncol. 23(4), 1057-1063 (2016).

46. Eckardt J, Licht PB. Thoracoscopic or open surgery for pulmonary metastasectomy: an observer blinded study. Ann. Thorac. Surg. 98(2), 466-469 (2014). 
47. Guerrera F, Renaud S, Schaeffer M et al. Low accuracy of computed tomography and positron emission tomography to detect lung and lymph node metastases of colorectal cancer. Ann. Thorac. Surg. 104(4), 1194-1199 (2017).

48. Robert JH, Ambrogi V, Mermillod B, Dahabreh D, Goldstraw P. Factors influencing long-term survival after lung metastasectomy. Ann. Thorac. Surg. 63(3), 777-784 (1997).

49. Abecasis N, Cortez F, Bettencourt A, Costa CS, Orvalho F, de Almeida JM. Surgical treatment of lung metastases: prognostic factors for long-term survival. J. Surg. Oncol. 72(4), 193-198 (1999).

50. Leo F, Cagini L, Rocmans P et al. Lung metastases from melanoma: when is surgical treatment warranted? Br. J. Cancer 83(5), 569-572 (2000).

51. Friedel G, Pastorino U, Ginsberg RJ et al. Results of lung metastasectomy from breast cancer: prognostic criteria on the basis of 467 cases of the International Registry of Lung Metastases. Eur. J. Cardiothorac. Surg. 22(3), 335-344 (2002).

52. Piltz S, Meimarakis G, Wichmann MW, Hatz R, Schildberg FW, Fuerst H. Long-term results after pulmonary resection of renal cell carcinoma metastases. Ann. Thorac. Surg. 73(4), 1082-1087 (2002).

53. Planchard D, Soria JC, Michiels $S$ et al. Uncertain benefit from surgery in patients with lung metastases from breast carcinoma. Cancer 100(1), 28-35 (2004).

54. Vogelsang H, Haas S, Hierholzer C, Berger U, Siewert JR, Prauer H. Factors influencing survival after resection of pulmonary metastases from colorectal cancer. Br. J. Surg. 91(8), 1066-1071 (2004).

55. Shiono $\mathrm{S}$, Okumura $\mathrm{T}$, Boku $\mathrm{N}$ et al. Outcomes of segmentectomy and wedge resection for pulmonary metastases from colorectal cancer. Eur. J. Cardiothorac. Surg. 51(3), 504-510 (2017).

56. Casali C, Stefani A, Storelli E, Morandi U. Prognostic factors and survival after resection of lung metastases from epithelial tumours. Interact. Cardiovasc. Thorac. Surg. 5(3), 317-321 (2006).

57. Furak J, Trojan I, Szoke T, Tiszlavicz L, Eller J, Lazar G. Visceral pleural infiltration as a negative prognostic factor in lung metastasis. Interact. Cardiovasc. Thorac. Surg. 6(2), 196-199 (2006).

58. Harting MT, Blakely ML, Jaffe $\mathrm{N}$ et al. Long-term survival after aggressive resection of pulmonary metastases among children and adolescents with osteosarcoma. J. Pediatr. Surg. 41(1), 194-199 (2006).

59. Rena O, Papalia E, Oliaro A et al. Pulmonary metastases from epithelial tumours: late results of surgical treatment. Eur. J. Cardiothorac. Surg. 30(2), 217-222 (2006).

60. Veronesi G, Petrella F, Leo F et al. Prognostic role of lymph node involvement in lung metastasectomy. J. Thorac. Cardiovasc. Surg. 133(4), 967-972 (2007).

61. Winter H, Meimarakis G, Hoffmann G et al. Does surgical resection of pulmonary metastases of head and neck cancer improve survival? Ann. Surg. Oncol. 15(10), 2915-2926 (2008).

62. Lin BR, Chang TC, Lee YC, Lee PH, Chang KJ, Liang JT. Pulmonary resection for colorectal cancer metastases: duration between cancer onset and lung metastasis as an important prognostic factor. Ann. Surg. Oncol. 16(4), 1026-1032 (2009).

63. Rama N, Monteiro A, Bernardo JE, Eugenio L, Antunes MJ. Lung metastases from colorectal cancer: surgical resection and prognostic factors. Eur. J. Cardiothorac. Surg. 35(3), 444-449 (2009).

64. Takakura Y, Miyata Y, Okajima M, Okada M, Ohdan H. Short disease-free interval is a significant risk factor for intrapulmonary recurrence after resection of pulmonary metastases in colorectal cancer. Colorectal. Dis. 12(7 Online), e68-e75 (2010).

65. Borasio P, Gisabella M, Bille A et al. Role of surgical resection in colorectal lung metastases: analysis of 137 patients. Int. J. Colorectal. Dis. 26(2), 183-190 (2011).

66. Riquet M, Foucault C, Cazes A et al. Pulmonary resection for metastases of colorectal adenocarcinoma. Ann. Thorac. Surg. 89(2), 375-380 (2010).

67. Sardenberg RAdS, Figueiredo LPd, Haddad FJ, Gross JL, Younes RN. Pulmonary metastasectomy from soft tissue sarcomas. Clinics 65(9), 871-876 (2010).

68. Tacconi F, Ambrogi V, Pompeo E, Sellitri F, Mineo TC. Substernal hand-assisted videothoracoscopic lung metastasectomy: long term results in a selected patient cohort. Thor. Cancer 2(2), 45-53 (2011).

69. Casiraghi M, De Pas T, Maisonneuve P et al. A 10-year single-center experience on 708 lung metastasectomies: the evidence of the "international registry of lung metastases". J. Thor. Oncol. 6(8), 1373-1378 (2011).

70. Long H, Zheng Y, Situ D, Ma G, Lin Z, Wang J. Hand-assisted thoracoscopic surgery for bilateral lung metastasectomy through sternocostal triangle access. Ann. Thorac. Surg. 91(3), 852-858 (2011).

71. Stephens EH, Blackmon SH, Correa AM et al. Progression after chemotherapy is a novel predictor of poor outcomes after pulmonary metastasectomy in sarcoma patients. J. Am. Coll. Surg. 212(5), 821-826 (2011).

72. Zabaleta J, Aguinagalde B, Fuentes MG et al. Survival after lung metastasectomy for colorectal cancer: importance of previous liver metastasis as a prognostic factor. Eur. J. Surg. Oncol. 37(9), 786-790 (2011).

73. Chua TC, Scolyer RA, Kennedy CW, Yan TD, McCaughan BC, Thompson JF. Surgical management of melanoma lung metastasis: an analysis of survival outcomes in 292 consecutive patients. Ann. Surg. Oncol. 19(6), 1774-1781 (2012). 
74. Hirosawa T, Itabashi M, Ohnuki $\mathrm{T}$ et al. Prognostic factors in patients undergoing complete resection of pulmonary metastases of colorectal cancer: a multi-institutional cumulative follow-up study. Surg. Today 43(5), 494-499 (2013).

75. Lo Faso F, Solaini L, Lembo R et al. Thoracoscopic lung metastasectomies: a 10-year, single-center experience. Surg. Endosc. 27(6), 1938-1944 (2013).

76. Cho JH, Hamaji M, Allen MS et al. The prognosis of pulmonary metastasectomy depends on the location of the primary colorectal cancer. Ann. Thorac. Surg. 98(4), 1231-1237 (2014).

77. Renaud S, Alifano M, Falcoz PE et al. Does nodal status influence survival? Results of a 19-year systematic lymphadenectomy experience during lung metastasectomy of colorectal cancer. Interact. Cardiovasc. Thorac. Surg. 18(4), 482-487 (2014).

78. Renaud S, Falcoz PE, Alifano M et al. Systematic lymph node dissection in lung metastasectomy of renal cell carcinoma: an 18 years of experience. J. Surg. Oncol. 109(8), 823-829 (2014).

79. Zampino MG, Maisonneuve P, Ravenda PS et al. Lung metastases from colorectal cancer: analysis of prognostic factors in a single institution study. Ann. Thorac. Surg. 98(4), 1238-1245 (2014).

80. Seebacher G, Decker S, Fischer JR, Held M, Schafers HJ, Graeter TP. Unexpected lymph node disease in resections for pulmonary metastases. Ann. Thorac. Surg. 99(1), 231-236 (2015).

81. Guerrera F, Mossetti C, Ceccarelli M et al. Surgery of colorectal cancer lung metastases: analysis of survival, recurrence and re-surgery. $J$. Thorac. Dis. 8(7), 1764-1771 (2016).

82. Franzke K, Natanov R, Zinne N et al. Pulmonary metastasectomy - a retrospective comparison of surgical outcomes after laser-assisted and conventional resection. Eur. J. Surg. Oncol. 43(7), 1357-1364 (2017).

83. Girelli L, Locati L, Galeone C et al. Lung metastasectomy in adenoid cystic cancer: is it worth it? Oral. Oncol. 65, 114-118 (2017).

84. Okiror L, Peleki A, Moffat D et al. Survival following pulmonary metastasectomy for sarcoma. Thorac. Cardiovasc. Surg. 64(2), 146-149 (2016).

85. Shiomi K, Naito M, Sato T et al. Effect of adjuvant chemotherapy after pulmonary metastasectomy on the prognosis of colorectal cancer. Ann. Med. Surg. (Lond.) 20, 19-25 (2017).

86. Sun F, Chen L, Shi M et al. Prognosis of video-assisted thoracoscopic pulmonary metastasectomy in patients with colorectal cancer lung metastases: an analysis of 154 cases. Int. J. Colorectal Dis. 32(6), 897-905 (2017).

87. Ricardi U, Filippi AR, Guarneri A et al. Stereotactic body radiation therapy for lung metastases. Lung Cancer 75(1), 77-81 (2012).

88. Siva S, Kirby K, Caine $\mathrm{H}$ et al. Comparison of single-fraction and multi-fraction stereotactic radiotherapy for patients with 18F-fluorodeoxyglucose positron emission tomography-staged pulmonary oligometastases. Clin. Oncol. 27(6), 353-361 (2015).

89. Aoki M, Hatayama Y, Kawaguchi $\mathrm{H}$ et al. Stereotactic body radiotherapy for lung metastases as oligo-recurrence: a single institutional study. J. Radiat. Res. 57(1), 55-61 (2016).

90. Binkley MS, Trakul N, Jacobs LR et al. Colorectal histology is associated with an increased risk of local failure in lung metastases treated with stereotactic ablative radiation therapy. Int. J. Radiat. Oncol. Biol. Phys. 92(5), 1044-1052 (2015).

91. Jung J, Song SY, Kim JH et al. Clinical efficacy of stereotactic ablative radiotherapy for lung metastases arising from colorectal cancer. Radiat. Oncol. 10, 238 (2015).

92. De Rose F, Cozzi L, Navarria P et al. Clinical outcome of stereotactic ablative body radiotherapy for lung metastatic lesions in non-small cell lung cancer oligometastatic patients. Clin. Oncol. 28(1), 13-20 (2016).

93. Helou J, Thibault I, Poon I et al. Stereotactic ablative radiation therapy for pulmonary metastases: histology, dose, and indication matter. Int. J. Radiat. Oncol. Biol. Phys. 98(2), 419-427 (2017).

94. Yamashita H, Niibe Y, Yamamoto $\mathrm{T}$ et al. Lung stereotactic radiotherapy for oligometastases: comparison of oligo-recurrence and sync-oligometastases. Jpn. J. Clin. Oncol. 46(7), 687-691 (2016).

95. Franceschini D, Cozzi L, De Rose F et al. Role of stereotactic body radiation therapy for lung metastases from radio-resistant primary tumours. J. Cancer Res. Clin. Oncol. 143(7), 1293-1299 (2017).

96. Ricco A, Davis J, Rate W et al. Lung metastases treated with stereotactic body radiotherapy: the RSSearch(R) patient Registry's experience. Radiat. Oncol. 12(1), 35 (2017).

97. Qiu H, Katz AW, Chowdhry AK et al. Stereotactic body radiotherapy for lung metastases from colorectal cancer: prognostic factors for disease control and survival. Am. J. Clin. Oncol. 41(1), 53-58 (2018). 